\title{
Surface forces and friction tuned by thermo-responsive polymer films
}

\author{
Moghaddam, Saeed Zajforoushan; Thormann, Esben
}

Published in:

Current Opinion in Colloid \& Interface Science

Link to article, DOI:

10.1016/j.cocis.2019.12.002

Publication date:

2020

Document Version

Peer reviewed version

Link back to DTU Orbit

Citation (APA):

Moghaddam, S. Z., \& Thormann, E. (2020). Surface forces and friction tuned by thermo-responsive polymer films. Current Opinion in Colloid \& Interface Science, 47, 27-45. https://doi.org/10.1016/j.cocis.2019.12.002

\section{General rights}

Copyright and moral rights for the publications made accessible in the public portal are retained by the authors and/or other copyright owners and it is a condition of accessing publications that users recognise and abide by the legal requirements associated with these rights.

- Users may download and print one copy of any publication from the public portal for the purpose of private study or research.

- You may not further distribute the material or use it for any profit-making activity or commercial gain

- You may freely distribute the URL identifying the publication in the public portal

If you believe that this document breaches copyright please contact us providing details, and we will remove access to the work immediately and investigate your claim. 


\section{Surface Forces and Friction Tuned by Thermo-Responsive Polymer}

\section{Films}

Saeed Zajforoushan Moghaddam and Esben Thormann*

Department of Chemistry, Technical University of Denmark, 2800 Kgs. Lyngby, Denmark 


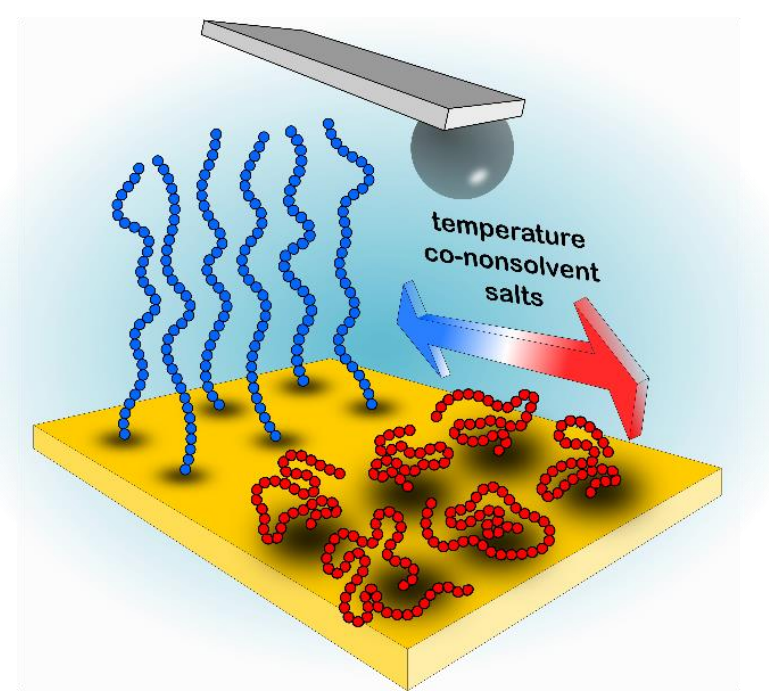

Abstract. Thermo-responsive polymer films have enabled the development of various functional surfaces with switchable interfacial properties. Assessing the surface forces and friction on such films is of paramount importance. On the one hand, it allows us to extract a great deal of information on the interfacial properties of the films, e.g., adhesiveness and lubricity, and how they could be tuned using different stimuli. On the other hand, surface force measurements complement other thin-film analysis methods, e.g., ellipsometry, to better perceive the correlation between the molecular properties of the polymer chains and the interfacial properties of the film. On this basis, we will, herein, provide a concise review of some recent studies on surface forces and friction tuned by thermo-responsive polymer films. This outline comprises a summary of several research works addressing the effects of temperature, solvent composition, and salts on surface forces and friction. In the end, we briefly discuss a few select studies in which the regulation of surface forces by thermo-responsive polymers is examined with an emphasis on the potential applications.

Keywords: thermo-responsive polymer, atomic force microscopy, surface forces, friction, colloidal probe 


\section{Introduction}

Thermo-responsive polymers belong to the large family of stimuli-responsive or smart polymers that are sensitive to changes in their environment.[1,2] Despite the extensive research conducted during the past few decades, the interest in thermo-responsive polymers continues to grow owing to the progressive developments in the design of new molecular structures and the tuning of their physicochemical properties.[3,4] Temperature, as an external physical stimulus, can induce a reversible phase transition, which is accompanied by variations in the conformation and interaction of the polymer. Such an "onoff' switchable control of the micro- and macroscopic properties of polymers offers vast application possibilities. Heat-triggerable polymeric vesicles (polymersomes) have been widely investigated as promising candidates for application in drug delivery, diagnostic imaging, therapeutic nanoreactors, and artificial organelles.[5,6] Herein, the assembly/disassembly of the polymer at temperatures close to the physiological body temperature controls the permeability and compound release. The biomedical applications of thermo-responsive polymers are not limited to vesicle systems; they have also been utilized to develop nano/microfibers as scaffolds for wound dressings, as well as culture substrates for cell harvesting.[7] For instance, the adhesion between the substrate and the cells can be modulated by varying the temperature; hence, one can control the cell morphology and attachment. Besides biomedical applications, thermo-responsive polymers have been vastly utilized to fabricate smart surfaces with controlled antifouling,[8] molecular separation,[9] bacterial adhesion,[10] and lubricity.[11]

Thermo-responsive polymers display a change in water miscibility/solubility, which is generally characterized by either an upper critical solution temperature (UCST, heating causes dissolution) or a lower critical solution temperature (LCST, heating causes phase separation). The LCST phase behavior is more prevalent between the commonly studied thermo-responsive polymers,[7] e.g., N-substituted poly(meth)acrylamides, poly(ethers), and poly(2-alkyl-2-oxazoline)s, which is why we, herein, focus on this system. Figure 1(a) illustrates the typical phase diagram (type II, i.e., a single minima) of a thermo- 
responsive polymer with LCST behavior.[12,13] For a given polymer concentration, the polymer coil is found in a highly hydrated, swollen, and hydrophilic state below the phase transition (critical) temperature. Heating above the phase separation temperature results in enhanced hydrophobicity and dehydration, which promote conformational collapse as well as interchain aggregation. In general, the LCST behavior originates from the competition between the enthalpic gain by hydration of the hydrophilic regions of the polymer against the entropic penalty by the hydration of nonpolar regions due to the hydrophobic effect. The hydrated state below the critical temperature arises from the dominant enthalpic term, whereas the phase separation results from the weakened polymer-water interaction, the relatively strong polymer-polymer attraction, and the entropic gain by the release of water molecules from the hydrophobic hydration shell.
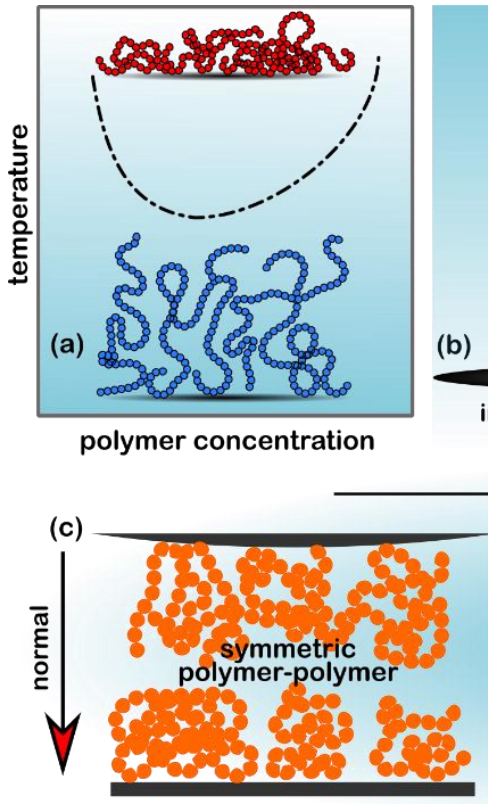
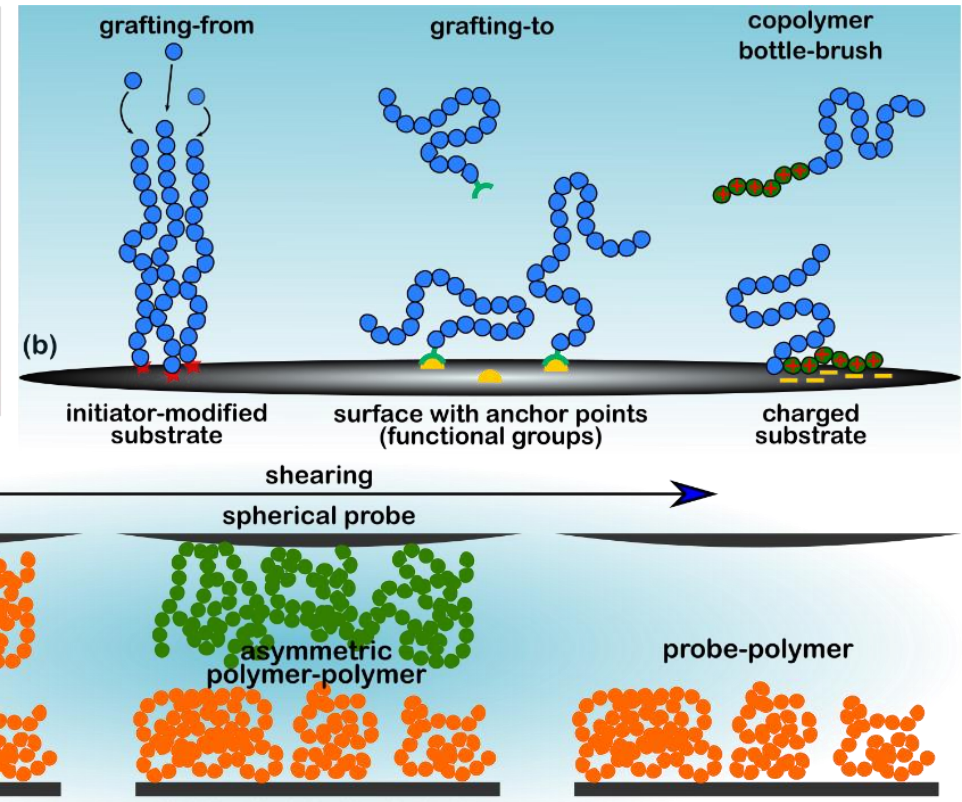

flat (underlying) substrate

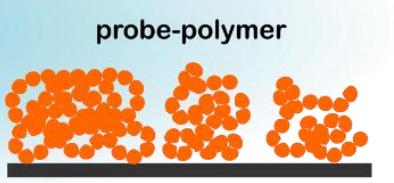

Figure 1 (a) Typical LCST phase diagram of a thermo-responsive polymer. The polymer chains adopt a swollen and hydrated state below the critical temperature, whereas a collapsed and hydrophobic state exists above the critical temperature. (b) Different approaches to attach the thermo-responsive polymers (units) to a solid substrate. The polymer is directly polymerized from the surface (grafting-from), adsorbed to the surface through specific interactions between the functional groups (grafting-to), or incorporated into a copolymer structure where the charged units promote adsorption to the charged substrate. (c) Atomic force microscopy (AFM) colloidal probe measurements on polymer-coated substrates in normal and shearing modes. 
Attaching the thermo-responsive polymer chains to a solid substrate is possible through different approaches (Figure 1(b)).[14,15] A common method involves the polymerization of the chains directly from the substrate decorated with initiator moieties, which is recognized as the grafting-from method.[16,17] Polymerization at the surface allows one to obtain relatively high grafting densities and produce extended polymer brush layers. Contrarily, thermo-responsive polymers functionalized with specific groups (either at one or multiple moieties) can be "grafted-to" favorable adsorption sites on the surface.[18,19] However, the steric hindrance between the polymer chains often prevents the achievement of relatively high grafting densities, thus affording less extended chains with a more flat conformation. As another common approach, the thermo-responsive units can be accommodated into a copolymer structure, e.g., block copolymers or bottle-brush polymers, composed of charged monomers that can adsorb onto an oppositely charged surface through electrostatic interactions.[20] The conformation of the chains at the surface is governed by various parameters, including the grafting density, bulkiness of the side chains, polymer-solvent, polymer-polymer, and polymer-surface interactions.[21] For instance, the polymer chains with a low grafting density form a "mushroom" conformation when monomer-solvent interactions prevail over monomer-substrate interactions, whereas strong interactions with the surface and a "bad solvent" condition can promote a flat "pancake" conformation. On the other hand, the balance of the repulsive excluded volume interactions against the unfavorable loss of configurational entropy of chain extension determines the degree of extension in a densely grafted polymer brush.

Polymer chains anchored to a substrate generally exhibit different phase transition characteristics compared to those of their "free" state in the bulk solution.[12] On the one hand, polymer chains confined to a solid surface usually undergo a broader collapse with increasing temperature compared to the rather sharp transition of non-grafted polymers.[22,23] On the other hand, the vertical collapse in terms of the variations in the polymer segment density is generally not uniform; instead, the polymer segment density shows a nonmonotonic gradient.[24,25] Accordingly, a polymer-rich region close to the 
substrate in equilibrium with dilute tails is a more accurate description than a uniform layer with a sharp interface. Consequently, the thermo-responsivity of polymer films, in terms of the variations of the bulk (interior) properties against those of the surface properties, could exhibit different trends. Therefore, one could expect discrepancies between the outcomes of the surface analysis methods sensitive to the periphery of the film (e.g., quartz crystal microbalance and atomic force microscopy (AFM)) against those sensitive to the dense interior region (e.g., ellipsometry and neutron reflectometry).[26-28] In general, it has been suggested that the bulk properties vary over a broad range of temperatures around the collapse temperature, whereas the surface properties vary over a relatively narrow range above the collapse temperature.[12] In addition, the phase transition behavior of the film, either in the interior or in the periphery zone, strongly depends on the hydration, molecular weight, and steric crowding.[12]

Different instrumental methods are available to assess the surface forces and friction on polymer-coated surfaces in micro- and macroscales.[29,30] AFM is a powerful tool that has been vastly employed to examine the interfacial properties of polymer surfaces. Besides topographical imaging, the colloidal probe technique has made it possible to examine the surface forces and friction on thermo-responsive polymer layers, and it is employed in most of the studies reviewed herein. Such force measurements (Figure 1(c)) are conducted not only between a bare colloidal probe and an underlying polymer-coated substrate but also between a probe and a substrate both bearing polymer layers (either symmetric or asymmetric). The former provides information on polymer-probe interactions, while the latter is concerned chiefly with polymer-polymer interactions. In addition, the force measurements are performed in normal and (or) shearing modes. In the former experiment, the probe approaches vertically and compresses into the surface until a defined maximum load is obtained, after which it is retracted. The latter experiment involves sliding the probe on the surface while a constant normal load is applied. The combination of these methods allows one to examine thermo-responsive polymer films in terms of structural variations, adhesiveness, viscoelasticity, and lubricity. Notably, the interaction between polymer layers has also been addressed in theoretical modeling and simulation studies; [31-34] however, we, herein, mainly focus on 
recent experimental findings. The surface forces between polymer-coated substrates in aqueous media have different origins, e.g., steric repulsion between the polymer chains, attractive bridging between the polymer and the probe, and interchain polymer interactions. The balance of these surface forces depends on multiple factors, including the solvent quality, thickness, grafting density, and molecular architecture. For instance, polymer-surface bridging occurs in the case of strong polymer-surface interaction and (or) poor solvent quality, where the tails can extend to the opposite surface, either bare or polymer-coated with a low grafting density. On the other hand, compressing polymer films together creates steric repulsive forces resulting from the entropic penalty upon confinement of the polymer chains between the surfaces, as well as the hydrodynamic forces associated with the expulsion of solvent from the compressed films.

In the following context, we provide a summary of the recent studies on force measurements conducted on thermo-responsive polymer films. As discussed earlier, the on-demand manipulation of the polymer conformation and interaction allows us to adjust the interfacial properties and surface forces of thermoresponsive films. Accordingly, the vast majority of the recent works focused on tuning the surface forces and friction on polymer layers by systematically varying the solvent quality through changing the temperature, solvent composition, as well as the addition of salts. Moreover, we present a few examples of recent studies with a more applied orientation, in which tuning the surface forces of thermo-responsive polymer layers is employed to develop smart surfaces with controlled adhesion to cells, minimized oil fouling, and selective adhesiveness to nanoparticles.

\section{Tunable Surface Forces and Friction}

\subsection{Temperature and Solvent Composition}

The most prevalent method to modulate the surface forces and friction of thermo-responsive films is through changing the temperature of the aqueous media.[35,36] Accordingly, surface force measurements are typically performed over temperatures ranging from below the transition temperature (good solvent 
condition), through the transition temperature, to well above the transition temperature (bad solvent condition). Another method for modifying the solvent quality involves maintaining a constant temperature while the solvent quality is systematically varied through the addition of a second solvent.[37,38] We outline some notable studies below that demonstrate how the transition from a swollen/hydrated state to a collapsed/hydrophobic state can affect the surface forces and friction.

$\mathrm{Yu}$ et al. conducted AFM force measurements between a bare gold probe and a poly $(\mathrm{N}$ isopropylacrylamide) (PNIPAM) brush at different temperatures between 20 and $40{ }^{\circ} \mathrm{C}$.[39] Thus far, we have highlighted that the interfacial properties of thermo-responsive polymer films typically vary between two extreme states below and above the critical temperature. On the contrary, this study suggests that the tribomechanical properties of the investigated PNIPAM brush evolve in a nonmonotonic manner with increasing temperature, with the maximal energy dissipation observed around the collapse temperature.

The force-distance profiles (Figure 2(a1)) provide a general insight into the conformation and interaction of the thermo-responsive brush at different temperatures. Below the collapse temperature, a purely repulsive approach force is observed, implying a highly swollen conformation that creates a large osmotic pressure. On the other hand, the retraction curve overlaps with the approach curve, suggesting a minor energy dissipation within the brush and a negligible polymer-probe adhesion. Therefore, compressing the probe against the hydrated polymer brush leads merely to solvent-squeeze-out. At temperatures near the transition temperature $\left(\sim 32^{\circ} \mathrm{C}\right)$, the repulsive force, upon approach, becomes relatively shortranged, which signifies a vertical collapse in the brush thickness. In addition, the retraction profile represents a long-range attractive interaction that hints to a strong polymer-probe adhesiveness, which results in stretching of the partly collapsed chains by the probe. At temperatures well above the transition temperature, the film thickness further collapses, while the adhesion becomes relatively short-ranged, showing a sharp snap-off adhesive force. Under this condition, one can argue that the polymer-polymer 
attractions, i.e., internal hydrogen bonding and hydrophobic interactions, within the film are strong enough to dominate the polymer-gold interaction. Consequently, the chains interacting with the gold surface resist stretching, which could explain the strong but short-range adhesive force. Accordingly, plotting the work of adhesion, i.e., the area between approach and retraction curves, against temperature, a maximum in energy dissipation is observed around the collapse temperature: a trend that is independent of the relative velocity and the applied normal load (Figure 2(a2)). The adhesion hysteresis below (solventsqueeze-out mechanism) and around (chain stretching mechanism) the collapse temperature exhibits a relatively strong dependence on both the velocity and the applied normal load, compared to the case of the fully collapsed state (snap-off mechanism), which affirms the changes in the viscoelastic nature of the film with increasing the temperature.
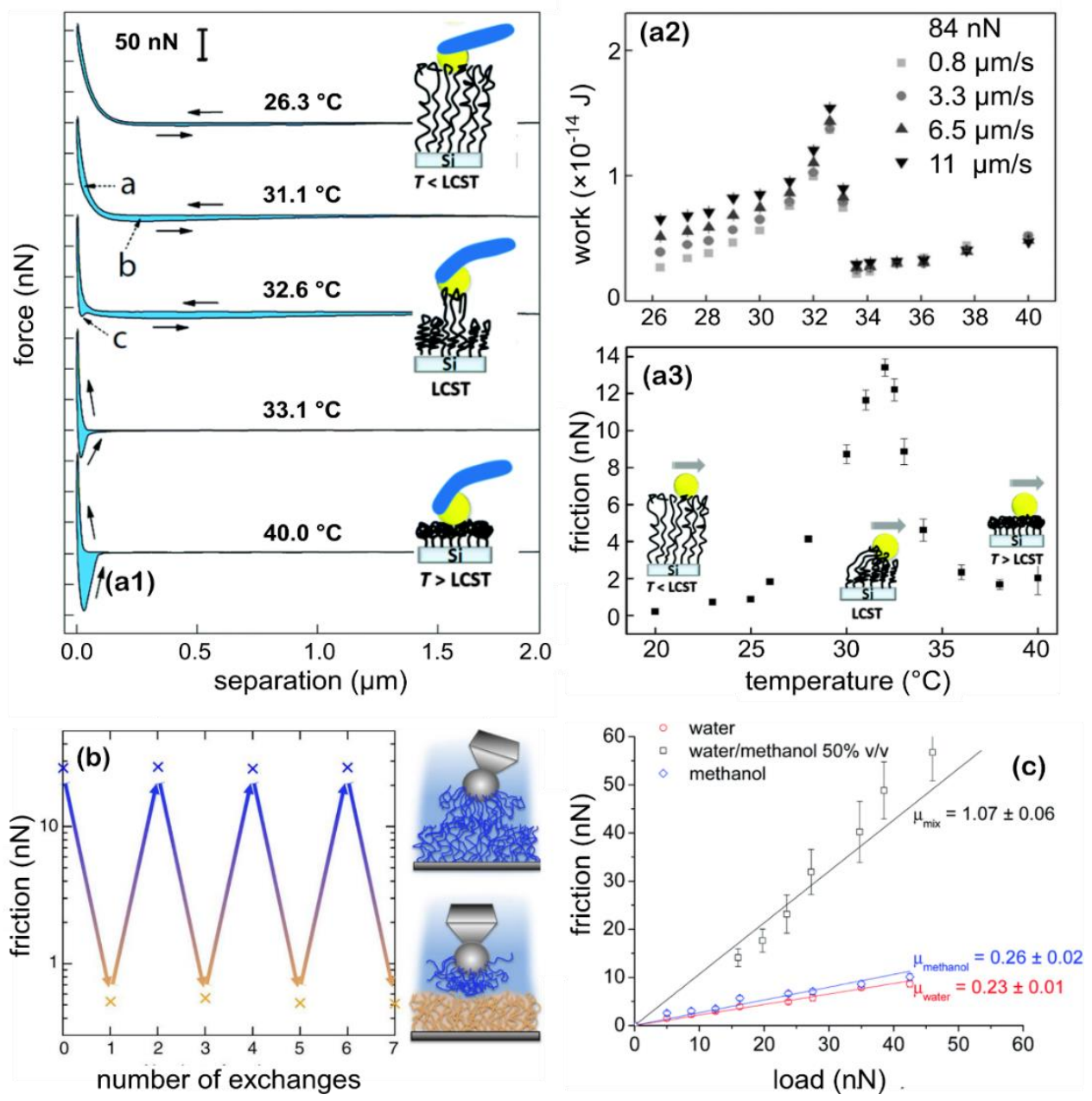
Figure 2 (panel a) Interfacial properties of a PNIPAM brush as a function of temperature (reprinted with permission from [39]); (a1) approach and retraction force-distance curves between a gold probe and a PNIPAM brush at different temperatures, (a2) adhesion hysteresis as a function of temperature at various velocities and a constant normal load of $84 \mathrm{nN}$, (a3) frictional forces measured as a function of temperature at a sliding velocity of $20 \mu \mathrm{m} \mathrm{s}^{-1}$ and a normal load of $2.2 \mathrm{nN}$. (b) Frictional force for the symmetric PNIPAM-PNIPAM brush system (top schematic) and the asymmetric PNIPAM-PMMA system (bottom schematic) at a velocity of $120 \mu \mathrm{m} \mathrm{s}^{-1}$ and a normal load of $180 \mathrm{nN}$ (reprinted with permission from [40]). (c) Frictional force-normal load plot for the PNIPAM brush (with medium grafting density) in pure and mixed solvents (reproduced with permission from [41]).

Friction measurements (Figure 2(a3)) also denote a significant energy dissipation around the collapse temperature. Accordingly, a small frictional force is obtained below the collapse temperature, which arises from the highly swollen state and minimum contact/adhesiveness between the probe and the brush. The frictional force monotonically grows with increasing the temperature in agreement with the gradual collapse and enhanced polymer-gold interaction. Around the collapse temperature, a considerably large frictional force is measured that confirms the stretching of the bridging chains between the sliding surfaces. A further increase in the temperature results in reduced frictional forces suggesting that the polymer chains resist stretching. Nevertheless, the frictional forces for the collapsed state are, in general, greater than those for the swollen state due to the polymer-gold adhesiveness. Thus, the balance of polymer-solvent, polymer-polymer, and polymer-probe interactions determines the surface forces at different temperatures. Polymer-probe interactions can result in energy dissipation in terms of chain stretching or sharp snap-off, while the breakage and reformation of polymer-polymer interactions could produce energy dissipative events within the film. The next work better elucidates how stickiness at the interface contributes to the friction.

De Beer et al. conducted friction measurements between asymmetric and symmetric polymer brush systems.[40] The symmetric system includes water-immersed PNIPAM brushes on both the probe and the surface, while the asymmetric system comprises a PNIPAM brush (on probe) against a poly(methyl methacrylate) (PMMA) brush (on silica wafer) pre-solvated in acetophenone (good solvent for PMMA). Both the systems are immersed in water to avoid the formation of an acetophenone capillary between the PNIPAM and PMMA brushes. As shown in Figure 2(b), sliding the symmetric brushes against each 
other produces significantly large friction, suggesting a substantial overlap and interpenetration of the polymer tails between the brushes. In contrast, two immiscible brushes provide a slick interface with notably low friction, implying minor interdigitation and, thus, energy dissipation. A similar lubrication behavior is observed when sliding asymmetric PNIPAM-PMMA brushes compared to the symmetric PMMA-PMMA system. Accordingly, one of the essential requirements for low friction is a sharp interface between the polymer-coated surfaces, which, herein, is provided by solvents that separate into two phases upon contact. The minimized polymer-polymer interpenetration at the interface not only provides lubrication but also decreases the possibility of film wear; thus, an optimal load-bearing capacity is achieved. It should be, however, noted that a slick interface cannot solely guarantee low friction as energy dissipative events could still occur within the film, which could be minimized by reducing the viscoelastic nature of the film.[42]

Switching the interfacial properties by heating and cooling might be impractical (considering the energy costs) when repeated "on-off” cycles are required. Instead, one can modulate the interfacial properties by changing the solvent composition. Chen et al. investigated the conformational transition and variations of the lubrication properties of PNIPAM films under good and bad solvent conditions.[41] The solvent quality, in the study, is adjusted through the systematic addition of methanol as a co-nonsolvent. The addition of methanol to PNIPAM in water leads to a collapsed state, whereas PNIPAM swells again beyond a particular methanol concentration. This swollen-collapsed-swollen transition is generally recognized as the re-entry transition, which is attributed to the balance between the solvent-solvent and solvent-polymer interactions. In a mixed solvent of water and methanol, the solvent-solvent interactions are stronger than the solvent-polymer interactions due to the formation of water-methanol complexes. This leads to a poor solvent quality, and thus, the polymer collapses.

Friction measurements between a silica probe and a PNIPAM brush in water, methanol, and the 50\% $\mathrm{v} / \mathrm{v}$ mixture reveal the significant effect of the solvent quality on the interfacial properties. In all cases, 
the frictional force-normal load curves (Figure 2(c)) display a linear trend (known as the Amontonian relationship) with negligible adhesion (nearly zero intercept value at zero applied load) and no indication of film wear up to normal loads of around $50 \mathrm{nN}$. Regarding the effect of solvent quality, the friction coefficients (dimensionless slope of the frictional force-load plot) in water and methanol are approximately similar, whereas it is almost five times larger in the mixed solvent. The elevated friction in the mixed solvent is ascribed to the enhanced polymer-probe affinity, which creates a sticky interface. Besides studying the effect of solvent quality, this work also highlights that a systematic increment in the grafting density of the brush produces significant friction under a given solvent condition, while such an effect is more pronounced under a bad solvent condition. For instance, increasing the grafting density from 0.03 to 0.69 chains. $\mathrm{nm}^{-2}$ leads to a twofold increment of the friction coefficient in pure water, whereas it causes an 8-fold increment in the mixed solvent.

This effect is probably due to the strong polymer-probe bridging and adhesion in the dense state. Accordingly, the surface forces and friction on thermo-responsive films strongly depend on the structural properties of the film, such as the grafting density and molecular architecture.[43-47] Since our review is chiefly focused on tuning the surface forces by varying the solvent condition, we do not discuss, in detail, how various structural parameters affect the interfacial properties of polymer films. An interested reader, however, is referred to the review studies by Benetti et al.[48] and Dédinaité et al.[49] for more thorough scrutiny of the topic. Shortly, we discuss a study describing how the film thickness together with the scanning distance could modify the friction profiles. 

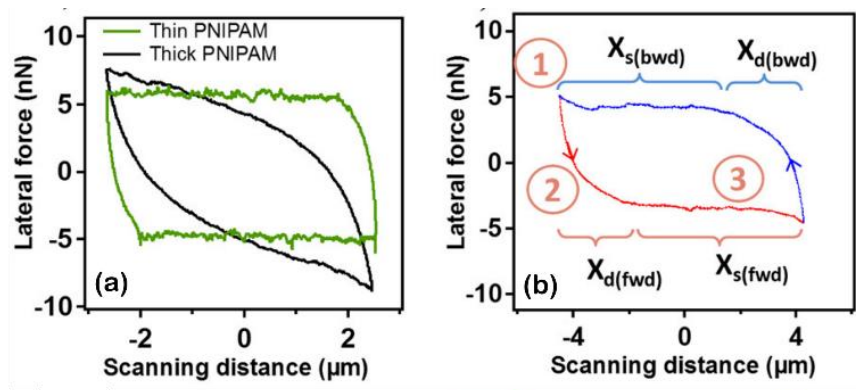

(c) (1)

(2) $x_{d}$

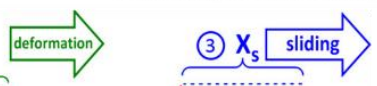

AFM probe

AFM probe 졸

AFM probe

AFM probe
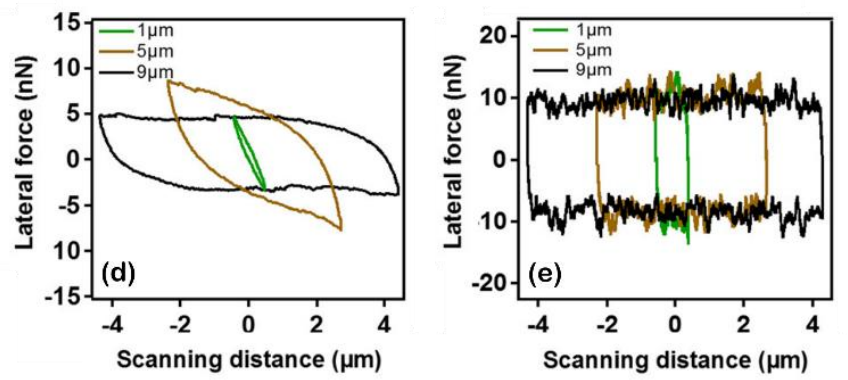

Figure 3 Effect of the polymer thickness and scanning distance on the friction profiles (reproduced with permission from [50]); (a) friction loops on thin and thick PNIPAM brushes in water at $25{ }^{\circ} \mathrm{C}$ for an applied load of $10 \mathrm{nN}$, (b) typical friction loop on thick PNIPAM brushes; the tilted portion ( $\mathrm{X}_{\mathrm{d}}$ ) and the sliding portion $\left(\mathrm{X}_{\mathrm{s}}\right.$ ) of the loop are highlighted, (c) schematic representation of brush deformation and sliding during lateral scanning, (d) friction loops recorded on thick PNIPAM brushes at $25{ }^{\circ} \mathrm{C}$ with scanning distances of 1,5 , and $9 \mu \mathrm{m}$, (e) frictional forces for thick PNIPAM brushes at $40{ }^{\circ} \mathrm{C}$.

Ramakrishna et al. investigated the effect of lateral deformation of PNIPAM brushes when measuring the frictional forces.[50] To do so, the friction loops (loading-unloading) obtained on thin $(\sim 10 \mathrm{~nm})$ and thick ( $500 \mathrm{~nm}$ ) brushes at $25{ }^{\circ} \mathrm{C}$ are compared (Figure 3(a)). Accordingly, the PNIPAM brushes, when swollen, both display tilted friction loops, which is attributed to the lateral deformation and stretching of the chains under shear force (Figures 3(b),(c)). However, this effect is more pronounced for the thick brush, i.e., the tilted portion of the friction trace $\left(X_{d}\right)$ is relatively larger. According to Figure 3(d), the friction loops on the swollen thick brush considerably depend on the applied scanning distance. Regarding the shortest scanning distance, the friction loop is entirely tilted, meaning that solely lateral deformation occurs without any sliding between the probe and the brush (static friction). A scanning distance of $5 \mu \mathrm{m}$ produces an initial lateral deformation, after which sliding occurs. A further increase in the scanning distance provides a friction loop with a tilted section, followed by steady sliding, meaning 
that the frictional dissipation is primarily dominated by the sliding. The lateral deformation of the brush, however, has a minor effect on the friction loops when the film is collapsed. According to Figure 3(e), the friction loops do not represent any tilted portion even for the shortest scanning distance, implying that collapsed PNIPAM chains (due to enhanced rigidity and strong cohesiveness within the film) resist lateral stretching. Accordingly, the measured interfacial properties (friction coefficient herein) depend not only on the solvent quality but also on the structural features (thickness) and experimental parameters (scanning distance). Next, we examine some studies addressing thermo-responsive polymer films other than brush systems.

Natural polymers including various polysaccharide derivatives also display thermo-sensitivity.[51] Thormann et al. investigated the surface forces and friction between hydrophobized silica surfaces coated by thermo-responsive methylcellulose.[52] Methylcellulose comprises nonpolar methyl and polar hydroxyl groups, which result in temperature-dependent miscibility with water. According to Figure 4 (panel a), frictional force-load profiles strongly depend on the temperature. Below the critical temperature (Figure 4(a1)), the frictional force is relatively small for applied loads below $4 \mathrm{nN}$, which indicates a minor energy dissipation and steric repulsion between the layers. However, the frictional force markedly increases with the normal load leading to film wear and a rather weak load-bearing ability. Similarly, the immediate unloading curve displays significantly high friction values. On the other hand, increasing the temperature to the critical point leads to a relatively high friction coefficient that can be attributed to the reduced solvent quality (Figure 4(a2)). Nevertheless, the film possesses a relatively high load-bearing capacity as no trace of wear (constant slope in the profile) is detected up to an applied load of $12 \mathrm{nN}$, while the unloading curve superimposes the loading curve. Accordingly, the reduced solvent quality, the strong polymer-surface interaction, and the strong cohesion within the layers prevent film wear under shear force. Since a weak attraction exists between the films in the hydrated state, the poor lubrication and load-bearing ability must pertain to the energy dissipation within the film rather than at 
the sliding surface. A weak polymer-surface interface could also be a source of energy dissipation and, consequently, elevated friction, which is plausible for the physically adsorbed methylcellulose films. Therefore, one requires a polymer layer firmly attached to the substrate while exposing hydrophilic groups toward the solution to attain lubricity together with increased load-bearing ability. Next, we discuss how electrostatic attraction could be employed as an alternative strategy to anchor thermoresponsive polymers to a charged substrate.
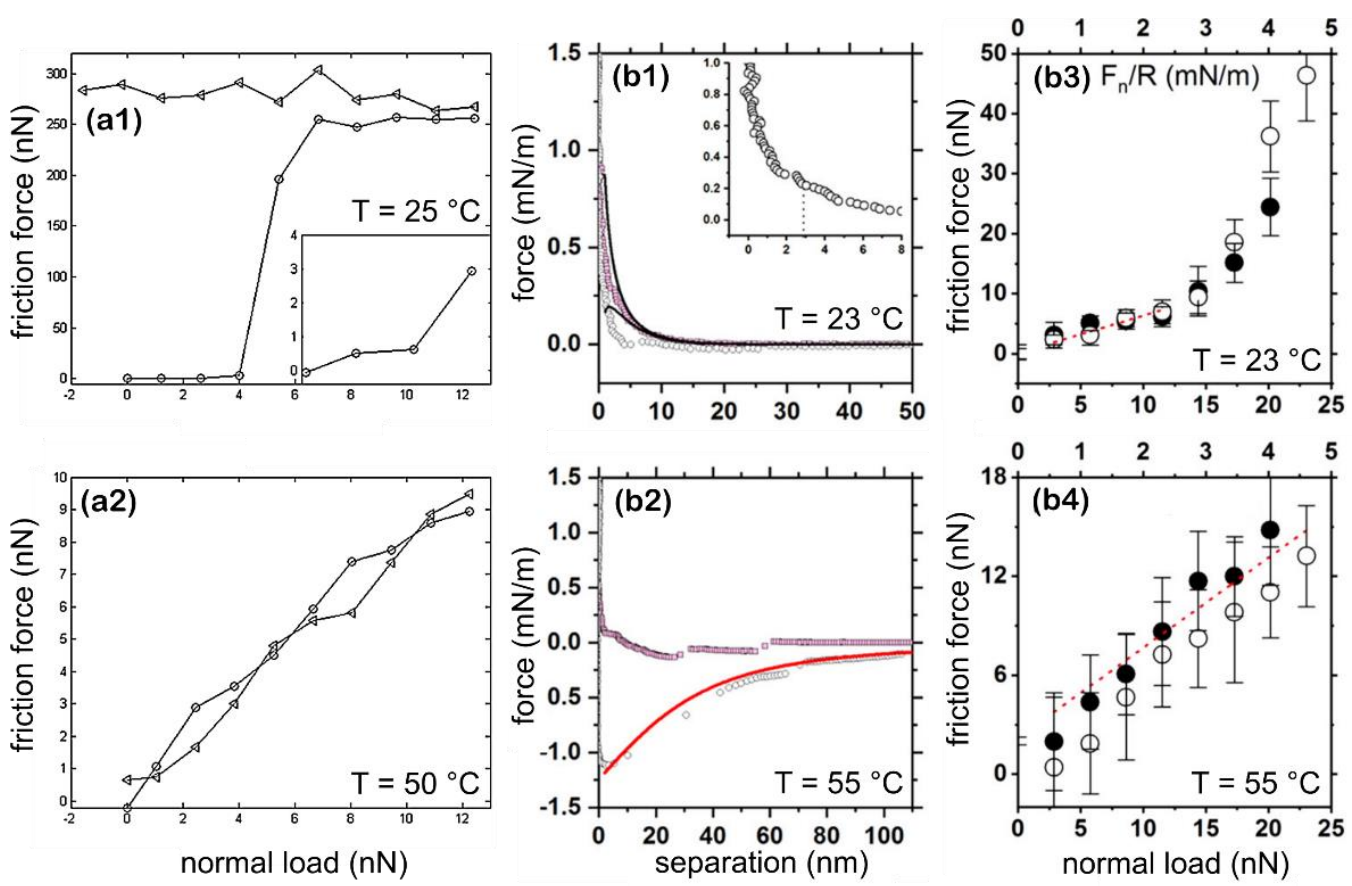

Figure 4 (panel a) Friction loops between methylcellulose films on hydrophobic silica surfaces at 25 (a1) and $50{ }^{\circ} \mathrm{C}$ (a2) (reproduced with permission from [52]). (panel b) Surface forces and friction between pentablock copolymer films on silica (reproduced with permission from [53]); force-distance profiles at 23 (b1) and $55^{\circ}(\mathrm{b} 2)$; friction loops at 23 (b3) and $55^{\circ} \mathrm{C}(\mathrm{b} 4)$.

Dobryden et al. examined the interfacial properties of a thermo-responsive pentablock copolymer electrostatically adsorbed to a silica surface.[53] The investigated copolymer has an $A-B-A-B-A$ structure, where A represents a cationic poly((3-acrylamido-propyl)-trimethylammonium chloride) (PAMPTMA) block, and B is an uncharged poly(2-isopropyl-2-oxazoline) (PIPOZ) block (collapse temperature $\sim 55^{\circ} \mathrm{C}$ ). Notably, the force measurements are conducted between the silica surfaces in the presence of the polymer solution. The force-distance profile below the critical temperature (Figure 4(b1)) 
comprises a short-range steric force $(\sim 3 \mathrm{~nm})$, which suggests a rather flattened conformation without long polymer loops or tails. Near the collapse temperature (Figure 4(b2)), a markedly long-range attractive jump-in is found when approaching, as well as a strong and long-range adhesion when retracting. The range of the adhesion force $(>100 \mathrm{~nm})$ is beyond the estimated length of the extended polymer chains, which rules out polymer bridging. Considering the shape of the adhesion force profile and the fact that the adhesion is considerably strong when the polymer is present in the solution, it is suggested that a capillary condensate of a polymer-rich phase forms in the gap between the surfaces.[35] As argued, the deposition of aggregates near the collapse temperature promotes the formation of the capillary condensate. With decreasing the surface separation, the free energy of the capillary phase also reduces due to a reduction of the volume of the capillary phase and the reduced interfacial area between the capillary and solution phases, which creates an attractive force between the surfaces.[54]

On the other hand, the friction profile below the critical temperature (Figure 4(b3)) displays a steady slope below the applied load of $12 \mathrm{nN}$, after which a marked increment in friction force (film wear) is observed. However, the friction profile at $55^{\circ} \mathrm{C}$ (Figure 4(b4)) indicates an enhanced load-bearing ability due to the poor solvent condition and strong PIPOZ-surface interaction. In addition, the friction coefficient is also smaller than that at low temperatures, which is unexpected considering the possible dissipative nature of the capillary condensate. The authors argue that the additional polymer adsorption at high temperatures could reduce the interpenetration of chains and, thus, lower the friction. Accordingly, while electrostatic anchoring could strengthen the attachment to the surface compared to the case in hydrophobically adsorbed systems, one can still expect a weak load-bearing capacity compared to that of covalently bound brush layers. However, the copolymer systems allow for fine-tuning the molecular architecture [55] and chemical composition [56] of the polymers, which offers various possibilities to fabricate films with controllable structures and interfacial properties. 
In a similar study, An et al. investigated the surface forces and friction between electrostatically adsorbed PIPOZ-PAMPTMA diblock copolymers at silica surfaces.[57] AFM colloidal probe measurements suggested a typical deterioration of the solvent quality manifested by the increased adhesion and friction with increasing temperature. To further verify this behavior, the authors calculated the interaction (free) energy between two surfaces bearing end-grafted PIPOZ layers as a function of surface separation using the lattice-mean field theory. The calculated interaction potential also demonstrated the typical features of solvent-quality deterioration with increasing temperature, i.e., the evolution of an attractive minimum at short separations, large interaction minimum, and a shift in the location of the minimum toward shorter separations. The model can also be employed to examine how the magnitude of the interaction minimum at different temperatures are affected by variations in the grafting density and the surface-polymer interaction parameter. To further verify the mechanisms behind this, the authors calculated the polymer volume fraction profiles. Accordingly, the fraction of penetrating polymer segments increases with increasing the interaction energy. In addition, for a given interaction energy, the degree of penetration increases with the temperature. Hence, the interpenetration of the chains is intensified as the solvent quality deteriorates under static conditions, which can also apply to sliding conditions. 

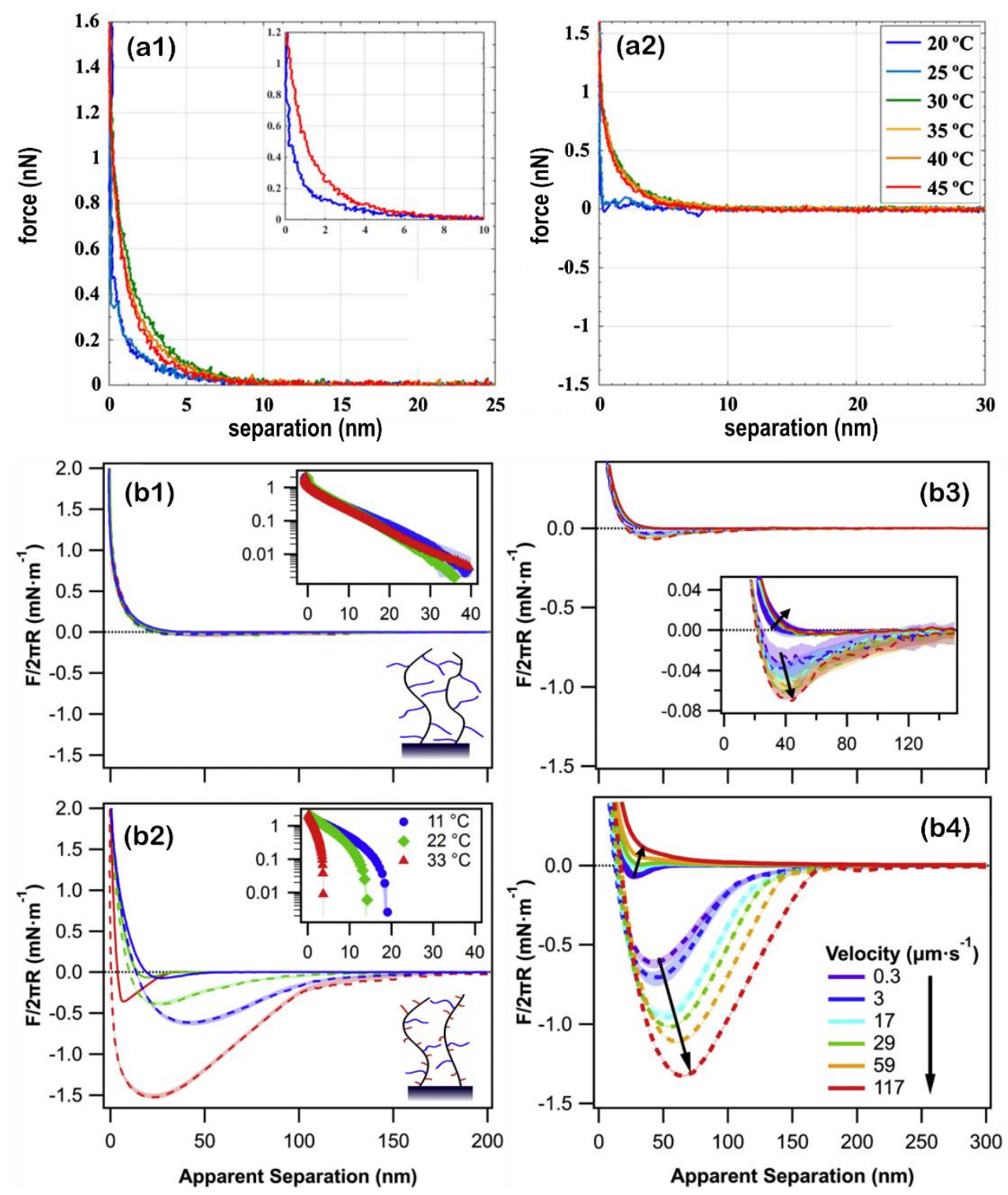

Figure 5 (panel a) Approach (a1) and retrace (a2) force-distance profiles between PEG-PNIPAM-PAMPTMA copolymer layers at the silica surface at temperatures between 20 and $45{ }^{\circ} \mathrm{C}$ (reproduced with permission from [58]). (panel b) Surface forces on ethylene glycol-based brushes at different temperatures and velocities (reproduced with permission from [59]); force-distance profiles between a bare silica probe and POEGMA300 (b1) and P(MEO2MA-stat-OEGMA300) (b2) brushes (approach: solid lines, retraction: dashed lines). Force-distance profiles between the bare probe and POEGMA300 (b3) and P(MEO2MA-stat-OEGMA300) (b4) brushes at different velocities at $11{ }^{\circ} \mathrm{C}$.

As discussed, the copolymer systems allow for molecular structure design and for consequent modulation of the interfacial properties. For instance, we investigated the surface forces between thermo-responsive triblock copolymer films on silica surfaces.[58] The studied triblock copolymer has an A-B-C structure, where A, B, and C represent poly(ethylene glycol) PEG, PNIPAM, and cationic PAMPTMA units, respectively. Accordingly, the charged blocks promote adsorption, the PNIPAM blocks could form an 
intermediate layer, while the PEG blocks should be found dangling on top. The force-distance measurements are conducted at temperatures between $20^{\circ} \mathrm{C}$ (where both PNIPAM and PEG blocks are hydrated) and $45{ }^{\circ} \mathrm{C}$ (where PEG blocks are expectedly hydrated; however, PNIPAM blocks must be collapsed). Regarding the approach step (Figure 5(a1)), one can observe purely repulsive force profiles at all temperatures. Along the same line, weak adhesion is observed when retracting at 20 and $25^{\circ} \mathrm{C}$, whereas no evident adhesion exists at elevated temperatures (Figure 5(a2)). As discussed earlier, the attractive forces between the PNIPAM segments around and above the critical temperature should cause relatively strong adhesion. Accordingly, the presence of PEG blocks at the film surface induces a shielding effect that prevents adhesion even if the PNIPAM blocks are collapsed. Moreover, while increasing the temperature has a minor effect on the onset of repulsion, the repulsive force increases in magnitude (at the same separation distance) at temperatures above $30{ }^{\circ} \mathrm{C}$. Therefore, while the outer PEG-rich layer appears to remain unaffected by heating, the PNIPAM blocks within the film undergo a conformational collapse to form a more compact and less compressible inner layer. Using such an approach, one can, thus, selectively regulate the thermo-responsive properties at the interface and within the film. Next, we show that one can tune the chemical composition of the polymer chains to modulate the thermoresponsivity and interfacial characteristics.

Murdoch et al. conducted a survey on ethylene glycol-based brushes, investigating the effect of copolymer composition on surface forces and thermo-responsivity.[59] To do so, two polymer brushes with slightly different chemical structures, i.e., a poly oligo(ethylene glycol) methyl ether methacrylate (POEGMA300) homopolymer and a P(MEO2MA-stat-OEGMA300) with $60 \mathrm{~mol} \%$ OEGMA300 and 40 mol\% (di(ethylene glycol) methyl ether methacrylate) (MEO2MA) statistical copolymer, are compared. The first important highlight of this work is that different surface analysis methods could predict different thermoresponsive properties. Herein, ellipsometry and neutron reflectometry measurements suggest that the POEGMA300 brush undergoes a monotonic decrease in thickness in the range of $10-70{ }^{\circ} \mathrm{C}$, while 
P(MEO2MA-stat-OEGMA300) shows a similar behavior, but with a steeper collapse. The force measurements (Figures 5(b1), (b2)) between a silica probe and the brushes $\left(0.3 \mu \mathrm{m} \mathrm{s}^{-1}\right.$ velocity) indicate apparently contradictory thermo-responsive behaviors. Accordingly, POEGMA300 displays a purely repulsive approach curve, while increasing the temperature has almost no effect on the onset of compression. As explained earlier, the AFM colloidal probe method is primarily sensitive to the periphery of the brush comprising the dilute tails. Conversely, methods such as ellipsometry are mostly sensitive to the variations in the dense bulk material. Therefore, the dilute tails are not included in the analysis model, and the layer is described as a film with a uniform density profile and a sharp cut off. In addition, AFM does not measure the absolute distances between the probe and the underlying substrate. Instead, it only measures the relative (apparent) distances set by the constant compliance region, which can lead to a bias in the interpretation of the zero-separation point and, thus, the range of interactions. On that basis, one can infer that the POEGMA300 brush undergoes a marked collapse in the dense inner regions, whereas the outer tails seem to remain swollen and hydrated. Besides, the retraction curves superimpose with the approach curves indicating a negligible hysteresis and a swollen brush state. In contrast, with increasing the temperature, the P(MEO2MA-stat-OEGMA300) brush demonstrates a decrease in the apparent thickness, as well as an enhanced hysteresis between the approach and retraction curves. The former suggests a relatively sharp brush-solution interface due to a more collapsed conformation, while the latter alludes to the bridging forces between the chains and the silica surface.

Besides the differences in thermo-responsivity and surface forces under quasi-equilibrium conditions, the brushes display distinguished viscoelastic responses as well. The force-distance measurements are performed at several velocities up to $117 \mu \mathrm{m} \mathrm{s}^{-1}$ to assess the hydrodynamic and viscoelastic effects (Figures 5(b3), (b4)). Accordingly, POEGMA300 shows minimal dependence on the relative velocity as only a small increment in adhesion during retraction is evident. Oppositely, the P(MEO2MA-statOEGMA300) brush demonstrates significant dependence on the relative velocity in both approach and 
retraction steps. Increasing the velocity weakens the attractive minima until $29 \mu \mathrm{m} \mathrm{s}^{-1}$, after which a purely repulsive profile is obtained, which could be attributed to the frequency-dependent apparent viscosity and solvent drainage. In addition, the reduced contact time upon increasing the velocity could explain the reduction in the attractive minimum and the diminished bridging. On the other hand, the adhesion during retraction progressively increases with the relative velocity, which could also be explained based on the hindered solvent drainage. Accordingly, an elevated adhesion is expected if the probe retracts faster than the solvent can return to the previously compressed area. According to this study, a slight modification to the molecular structure of the polymer can have significant effects on the surface forces, thermo-responsivity, and viscoelastic characteristics.

\subsection{Addition of Salts}

It is long known that the conformation and interactions of thermo-responsive polymers in aqueous media can be adjusted using salts.[60-62] This effect cannot be explained merely based on electrostatic forces; instead, it also depends on salt- or ion-specific dispersion and hydration forces. Accordingly, anions and cations are ranked according to their ability to stabilize or destabilize the polymer in the solution, which is widely recognized as the Hofmeister series. The effect of salts on the stability of thermo-responsive polymers is often quantified in terms of the shift in the collapse temperature as a function of the salt type and concentration (Figure 6(a)). Accordingly, a weakly hydrated salt, e.g., NaSCN, enhances the polymer stability (salting-in effect) and increases the phase separation temperature in a nonmonotonic manner. Conversely, a strongly hydrated salt, e.g., $\mathrm{NaF}$, causes a drop in the collapse temperature and deteriorates the solvent quality. According to common belief, weakly hydrated salts prefer the polymeric surface, while strongly hydrated salts are effectively depleted. Therefore, one can tune the properties of thermoresponsive polymer films at a fixed temperature only by changing the salt type or concentration. The surface forces on thermo-responsive polymers in saline solutions have been investigated to assess the 
interfacial properties of a given polymer film in the presence of salts and (or) to understand the fundamental mechanisms of the specific ion effects.[63,64]

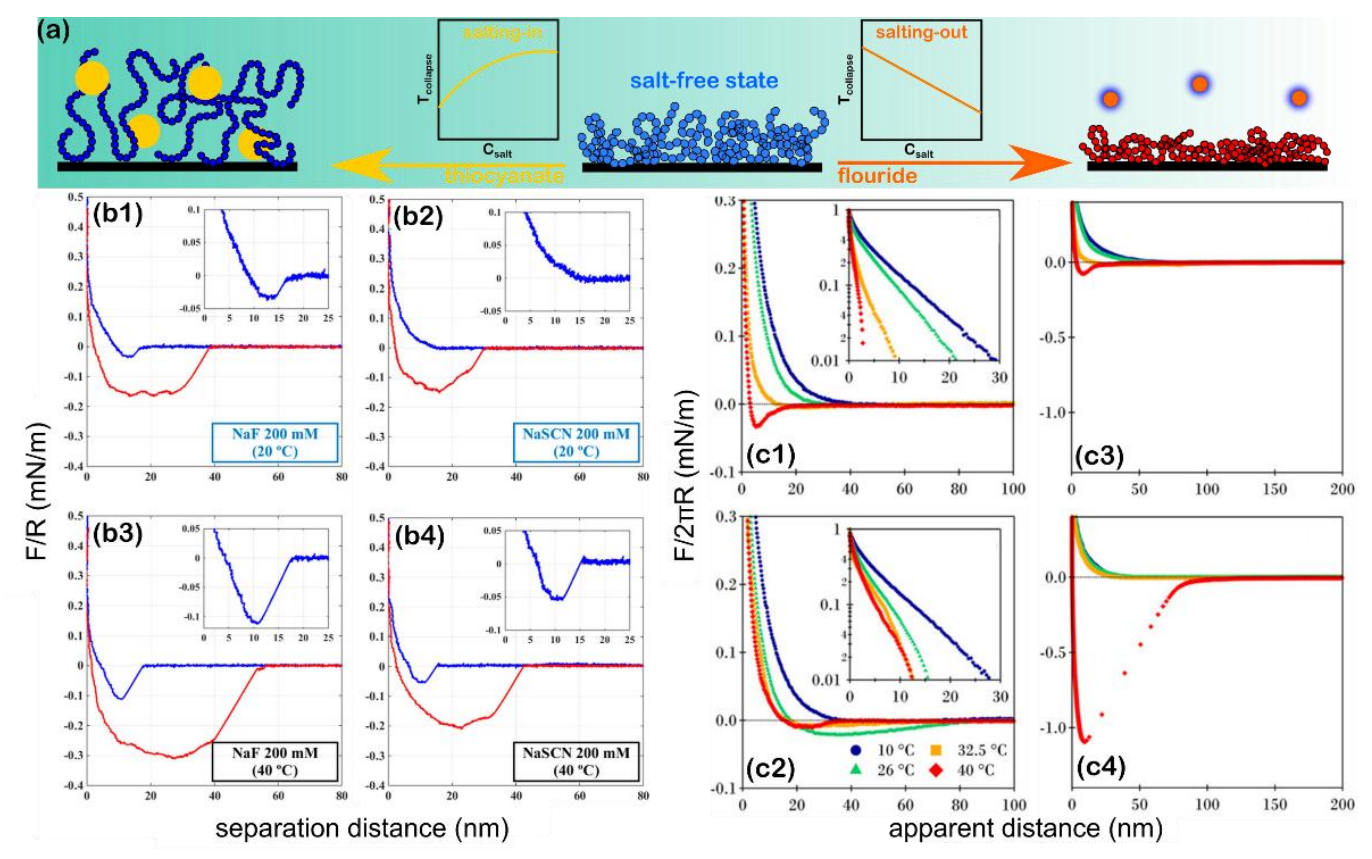

Figure 6 Effect of salts on thermo-responsive polymer films; (a) schematic illustration showing the collapse induced by strongly hydrated salts and swelling in the presence of weakly hydrated salts. (panel b) Force-distance profiles at 20 and $40{ }^{\circ} \mathrm{C}$ in the presence of $200 \mathrm{mM} \mathrm{NaF}$ and $\mathrm{NaSCN}$ obtained between gold surfaces coated by PNIPAM (reproduced with permission from [65]). (panel c) Approach (left) and retraction (right) force-distance profiles between a silica probe and a PNIPAM brush in $1 \mathrm{mM} \mathrm{KCl} \mathrm{(c1,} \mathrm{c3),} \mathrm{and} \mathrm{KSCN}$ (c2, c4) solutions at different temperatures. (reproduced with permission from [66]).

We investigated the surface forces between the PNIPAM films on gold surfaces in the presence of $\mathrm{NaSCN}$ and $\mathrm{NaF}$ at different temperatures (Figure 6, panel b).[65] It is evident that the force-distance profiles in $200 \mathrm{mM} \mathrm{NaSCN}$ differ significantly from those in $200 \mathrm{mM} \mathrm{NaF}$ solution. At $20^{\circ} \mathrm{C}$, when 200 $\mathrm{mM} \mathrm{NaF}$ is present (Figure 6 (b1)), the approach force curve comprises an attractive jump-in, followed by a repulsive steric compression. On retraction, strong adhesion is found in terms of both adhesion energy (hysteresis) and range. The strong adhesion, herein, suggests the presence of polymer-polymer and polymer-gold bridging forces. However, since the temperature is below the collapse point, we expect the latter to contribute more effectively. At $20{ }^{\circ} \mathrm{C}$, when $200 \mathrm{mM} \mathrm{NaSCN}$ is present (Figure 6 (b2)), a purely repulsive approach profile with no attractive jump-in is obtained; meanwhile, the retraction profile shows a relatively small adhesion energy. Considering that the addition of $\mathrm{NaSCN}$ enhances the solvent 
quality, one can expect weak attractive polymer-gold and polymer-polymer forces. Furthermore, the accumulation of the weakly hydrated salt within the film can promote a swollen conformation, which can sterically hinder the bridging of polymer chains to the opposing surface. The two salts have distinguished effects on the PNIPAM films even above the collapse temperature. At $40{ }^{\circ} \mathrm{C}$, in the NaF solution (Figure 6 (b3)), enhanced bridging and adhesion are observed, implying relatively strong PNIPAM-gold and PNIPAM-PNIPAM interactions. On the other hand, at $40^{\circ} \mathrm{C}$ in the $\mathrm{NaSCN}$ solution (Figure 6 (b4)), one can detect an attractive jump-in and gain in the adhesion energy (compared to the case at $20{ }^{\circ} \mathrm{C}$ ). While this observation affirms the collapse of the film, the adhesion is less pronounced compared to that in the $\mathrm{NaF}$ solution (almost 50\% smaller adhesion energy). Therefore, the weakly hydrated $\mathrm{NaSCN}$, through accumulation within the film, can improve the solvent quality and modulate the attractive forces even above the collapse temperature.

Humphreys et al. studied the effects of $\mathrm{KCl}$ and $\mathrm{KSCN}$ on PNIPAM brushes using two sets of surface force measurements.[66] First, force-distance profiles are measured between a bare silica probe and a PNIPAM brush grafted-from a flat silica surface in the presence of salts at different temperatures. Compared to the case in the presence of $1 \mathrm{mM} \mathrm{KCl}$ (Figure 6 (c1)), the approach profiles in the presence of $1 \mathrm{mM} \mathrm{KSCN}$ (Figure 6 (c2)) exhibit a more long-range attractive bridging and higher compressibility. The former observation suggests the presence of extended tails, whereas the latter finding refers to a less compact internal structure. Furthermore, the semi-log scale force-distance profiles (shown in inset) in the presence of KSCN display a slight deviation from linearity, which could suggest contributions from both steric and electrostatic repulsions due to the accumulation of ions within the film. Next, symmetric force-distance measurements are performed between two silica surfaces, both coated by PNIPAM brushes. Similarly, $\mathrm{KCl}$ promotes a stiff and collapsed brush state with favorable polymer-polymer interactions, whereas KSCN induces a soft swollen brush state and prevents hydrophobic brush-onbrush interactions. The interesting finding, herein, is the trend for adhesion on retraction at $40{ }^{\circ} \mathrm{C}$. Accordingly, the retraction profiles for $1 \mathrm{mM} \mathrm{KCl}$ (Figure 6(c3)) below and around the critical 
temperature present no evident adhesion. As expected for $40^{\circ} \mathrm{C}$, the retraction profile shows an attractive force between the collapsed hydrophobic brushes. The retraction profiles for $1 \mathrm{mM} \mathrm{KSCN}$ (Figure 6(c4)) at 10,26 , and $32.5^{\circ} \mathrm{C}$ also indicate no evident adhesion and purely steric interaction, whereas the retraction profile at $40{ }^{\circ} \mathrm{C}$ displays an unexpected and significantly strong adhesion (even stronger than that in the $\mathrm{KCl}$ solution). While this finding contradicts the expected stabilizing effect by KSCN, one can speculate that the adhesion originates from the specific binding of $\mathrm{SCN}^{-}$that induces bridging-like interactions between the polymer chains.

In another similar study, Murdoch et al. examined the effects of $\mathrm{KCH}_{3} \mathrm{COO}$ and $\mathrm{KSCN}$ on ethylene glycol-based thermo-responsive polymer brushes.[67] While most of the conclusions are similar to those of the previous work, the authors, herein, reported a longer-ranged repulsive compression in $\mathrm{KCH}_{3} \mathrm{COO}$ compared to that in a salt-free solution. This observation somehow contradicts the general expectation that the film should be more collapsed in $\mathrm{KCH}_{3} \mathrm{COO}$; nevertheless, it should be noted that the absolute separation between the surfaces is not afforded by AFM force measurements. Therefore, in this case, it is better to compare the ranges over which the attractive bridging interactions are observed. Accordingly, the approach profiles display a longer-range attraction in pure water than in $\mathrm{KCH}_{3} \mathrm{COO}$, which suggests the presence of extended tails that can interact with the probe. Similarly, the approach profiles in the KSCN solution show a long-range attractive bridging, implying the presence of dangling tails on the collapsed film.

In summary, salts modify polymer-polymer, polymer-surface, polymer-water, and water-water interactions below and above the critical temperature. Each of these interactions strongly depends on the type and amount of the present ions. In particular, weakly hydrated salts tend to accumulate within the polymeric film, which could induce specific interactions both within the film, e.g., crosslinking-like effect, and between the polymer films, e.g., bridging-like effect.

\section{Examples of Applications}


Thus far, we have discussed how different parameters such as temperature, solvent composition, and salts affect the interfacial properties and surface forces of thermo-responsive polymer films. The ability to modulate the surface forces and friction provides significant opportunities in designing smart functional surfaces. Herein, we have chosen three recent works in which the modulation of surface forces by thermo-responsive polymers is discussed from a practical point of view, as well. The first selected work utilizes the thermo-responsivity of PNIPAM as a tool to manipulate the adhesion of cells to their culture substrate. The second work focuses on how thermo-responsiveness and abrupt volume-phase transition of a PNIPAM copolymer could provide oil-repellant and self-cleaning properties. The last work describes how the adhesion forces on PNIPAM films can be switched "on" to catch synthesized nanoparticles from one solution and then switched "off" to release them in another solution. The discussions through these studies will provide a better perception of how closely the polymer conformation and interaction correlate to the surface forces.

Temperature-responsive cell culture substrates are promising tools in tissue engineering with potential application in the treatment/regeneration of human tissues and organs.[68,69] As a noninvasive and chemical-free harvest method, the thermally induced transition between the hydrophobic and hydrophilic states in PNIPAM-coated surfaces allows for the selective attachment and detachment of living cells, respectively. Besides temperature, the structural properties of the PNIPAM film such as the grafting density and thickness significantly affect the cell adhesion. It is suggested that relatively thick films demonstrate weak adhesiveness toward cells even above the collapse temperature, which is attributed to the presence of hydrated dilute tails that induce surface hydrophilicity. On the other hand, relatively thin films are also incapable of detaching the cells even below the collapse temperature, which is ascribed to the minor variations in the thickness and hydration. Based on this premise, Akiyama et al. fabricated a thermo-responsive cell culture substrate, comprising a PNIPAM brush grafted on a polydimethylsiloxane (PDMS) layer, which undergoes variations in thickness, surface wettability, and cell adhesion.[70] While 
the PNIPAM layer affords thermo-responsivity, the PDMS layer allows the stretching of the surface to reversibly alter the grafting density and thickness of the PNIPAM layer on top (Figure 7(a)). Forcedistance profiles (Figures 7(b1), (b2)) suggest that stretching the PDMS surface results in weak adhesiveness between the hydrophilic silicone probe and the PNIPAM film at $25{ }^{\circ} \mathrm{C}$. Thus, the mechanical stretching increases the hydrophobicity of the PNIPAM surface due to the reduced number of extended tails.

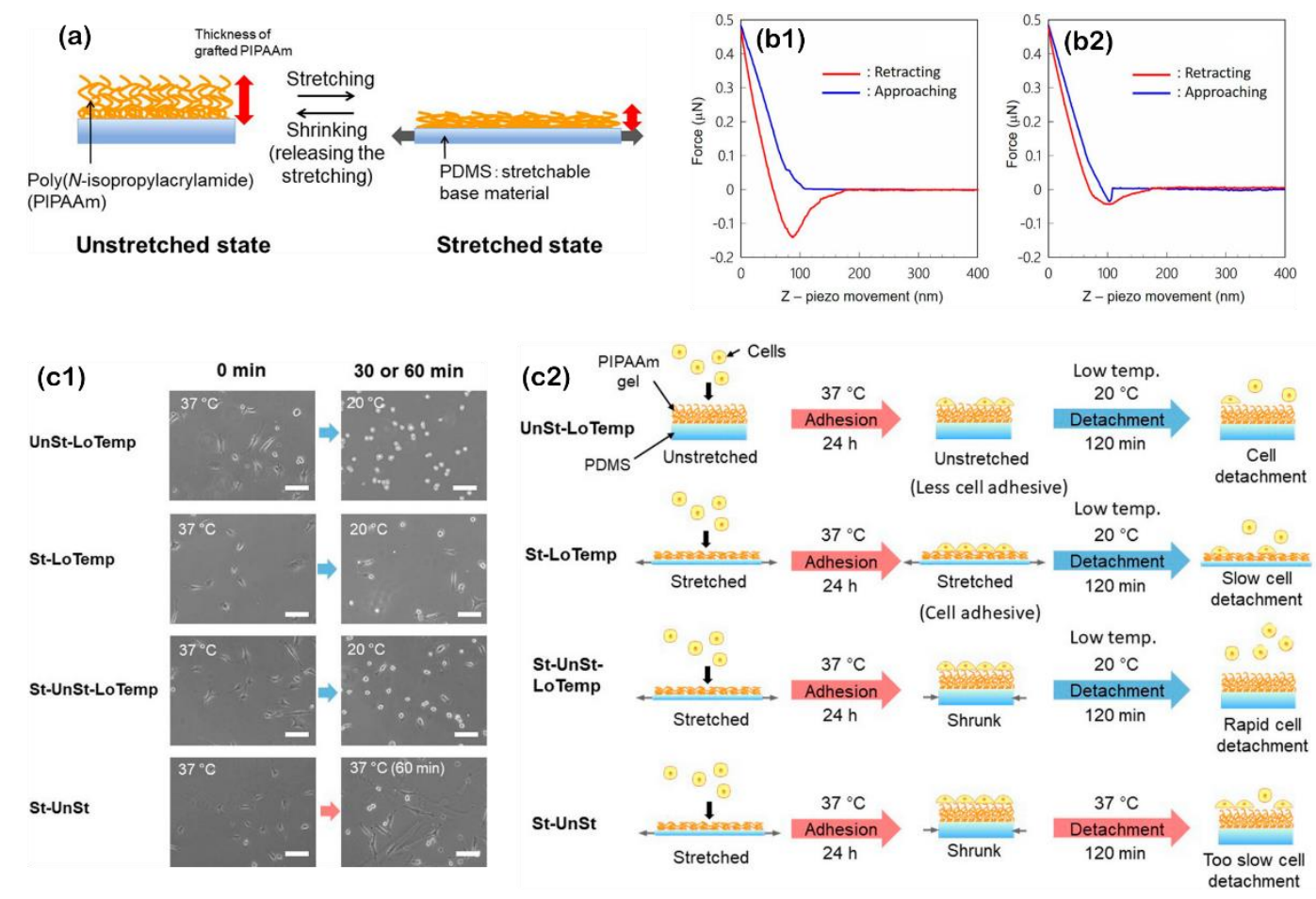

Figure 7 Thermo-responsive substrate for controlled cell attachment (reproduced with permission from [70]). (a) Schematic illustration of the engineered substrate. Stretching causes a decrease in the grafting density and thickness of the PNIPAM film. (panel b) Force-distance profiles obtained at $25{ }^{\circ} \mathrm{C}$ on unstretched (b1) and stretched (b2) substrates. (c1) Phase-contrast microscopic images of the cells before (left) and after (right) four different detachment procedures. (c2) Schematic illustration of four different detachment procedures showing the effect of temperature and mechanical stretching on cell adhesion.

Bovine aorta endothelial cells (BAECs) are cultured on the substrates and subsequently subjected to four different detachment procedures (Figure 7, panel c). Phase-contrast microscopic images and image analysis are used to assess the attachment/detachment of the cells in each case (Figure 7(c1)). The UnStLo'Temp procedure involves culturing the cells on the unstretched substrate at $37{ }^{\circ} \mathrm{C}$, followed by decreasing the temperature to $20^{\circ} \mathrm{C}$. St-LoTemp has the same procedure; however, the culturing occurs 
on the stretched substrate. The unstretched film provides less cell adhesion during the culturing as well as more cell detachment, which can both be attributed to the more hydrophilic surface with extended tails. St-UnSt-LoTemp involves seeding the cells on the stretched substrate at $37{ }^{\circ} \mathrm{C}$, followed by unstretching and decreasing the temperature to $20{ }^{\circ} \mathrm{C}$. This procedure entails dual stimulation and demonstrates the best cell detachment performance. Both the decrease in the temperature and unstretching promote surface hydrophilicity that favor cell detachment. The St-UnSt procedure involves seeding the cells on the stretched substrate at $37^{\circ} \mathrm{C}$, followed by unstretching while maintaining the temperature at $37^{\circ} \mathrm{C}$. A poor detachment performance is, hereon, observed, which implies that mechanical stress alone is ineffective and thermo-responsiveness is essential for cell detachment.

As the second example, Ye et al. fabricated thermo-responsive copolymer brushes of PNIPAM and poly(ethylene glycol) methacrylate (PEGMA) that display self-cleaning ability against underwater oil fouling.[71] Self-cleaning surfaces are typically low surface energy materials with quite large oil contact angles ( > 150); nevertheless, such surfaces exhibit significant adhesion to organic pollutants underwater. Thermo-responsive PNIPAM coatings could be an alternative solution for two reasons. On the one hand, the hydrophilic surface below the critical temperature promotes the desorption of the organic pollutant. On the other hand, the abrupt conformation change (in terms of thickness variation) during the transition could provide a strong mechanical stress or "washing force" to facilitate the self-cleaning process. However, the surface hydrophobicity of PNIPAM above the critical point is a disadvantage that favors oil adhesion. To address this issue, the surface hydrophobicity of PNIPAM is, herein, adjusted by incorporating small ratios of the hydrophilic PEGMA comonomer, which can provide a shielding effect for the hydrophobic PNPAM surface while preserving the sudden volume-phase transition.

Figure 8(a) shows a comparison of the adhesive forces required to separate an oil droplet from polymeric films at 25 and $50{ }^{\circ} \mathrm{C}$. Below the critical temperature, all the surfaces exhibit relatively small adhesive forces due to the surface hydrophilicity. The adhesive forces on the collapsed PNIPAM film at $50{ }^{\circ} \mathrm{C}$ are 
considerably stronger; nevertheless, increasing the PEGMA content in the copolymer reduces the adhesiveness. To evaluate the self-cleaning performance (Figures 8(b), (c)), the fouled surface is repeatedly rinsed in 25 and $50{ }^{\circ} \mathrm{C}$ water. PNIPAM (reference) and the copolymers with a low PEGMA content present poor efficiency in self-cleaning due to their surface hydrophobicity in the collapsed state. Contrarily, PEGMA (reference) and the copolymers with a high PEGMA content also leave rather large amounts of oil residue on the surface, due to the elevated collapse temperature and less abrupt volume transition. Thus, the copolymer with a PEGMA content of around $5 \mathrm{~mol} \%$ exhibits the best self-cleaning performance, due to the balanced surface hydrophilicity and the conformational transition.
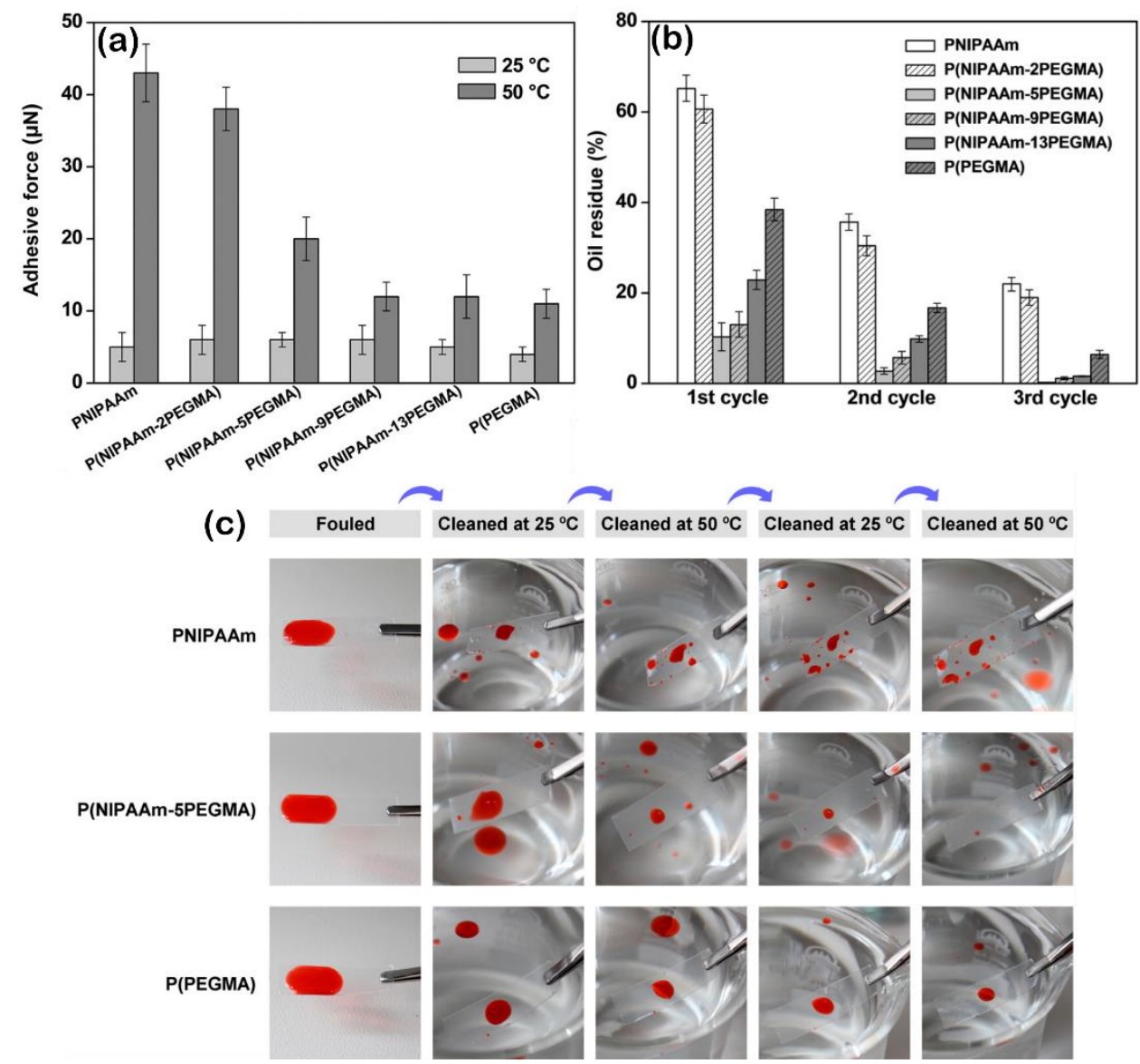

Figure 8 Thermo-responsive PNIPAM-PEGMA copolymer brush with self-cleaning capacity (reproduced with permission from [71]); (a) adhesive forces required to separate an oil droplet from the polymer brush surfaces at temperatures of 25 and $50^{\circ} \mathrm{C}$. (b) The amount of oil residues on the polymer brush surfaces after three cleaning cycles performed at 25 and $50{ }^{\circ} \mathrm{C}$. (c) The polymer brush surfaces fouled by oil and cleaned through three cycles. 
As the last example, Yu et al. employed co-nonsolvency of PNIPAM brushes to collect nanoparticles in a simple yet efficient manner without particle aggregation.[72] In the common preparation method, nanoparticles are synthesized in solution through hydrolysis and a polycondensation reaction, after which the particles must be transferred to another solvent for storage or further processing. The solvent exchange process is generally conducted by precipitating the particles and re-dispersing them in the new solution, which often results in aggregated particles that are difficult to re-disperse. In this work, the cononsolvency of PNIPAM is used to extract the nanoparticles from their solution and release them into another solution. As discussed earlier, the addition of short alcohols to PNIPAM in water gives rise to a swollen-to-collapsed-to-swollen transition. This means that in the mixed solvent, i.e., bad solvent condition, the polymer collapses and shows maximum hydrophobicity. Figure 9 (a) illustrates that the PNIPAM film exhibits significant adhesion (against bare silica probe) in a 30-70 vol\% ethanol-water mixture, whereas the adhesion is significantly weak in pure ethanol. Accordingly, the PNIPAM film is selectively (and reversibly) adhesive toward silica particles (also other particles such as gold, polystyrene, and PMMA) only by switching the solvent condition without changing the temperature. 

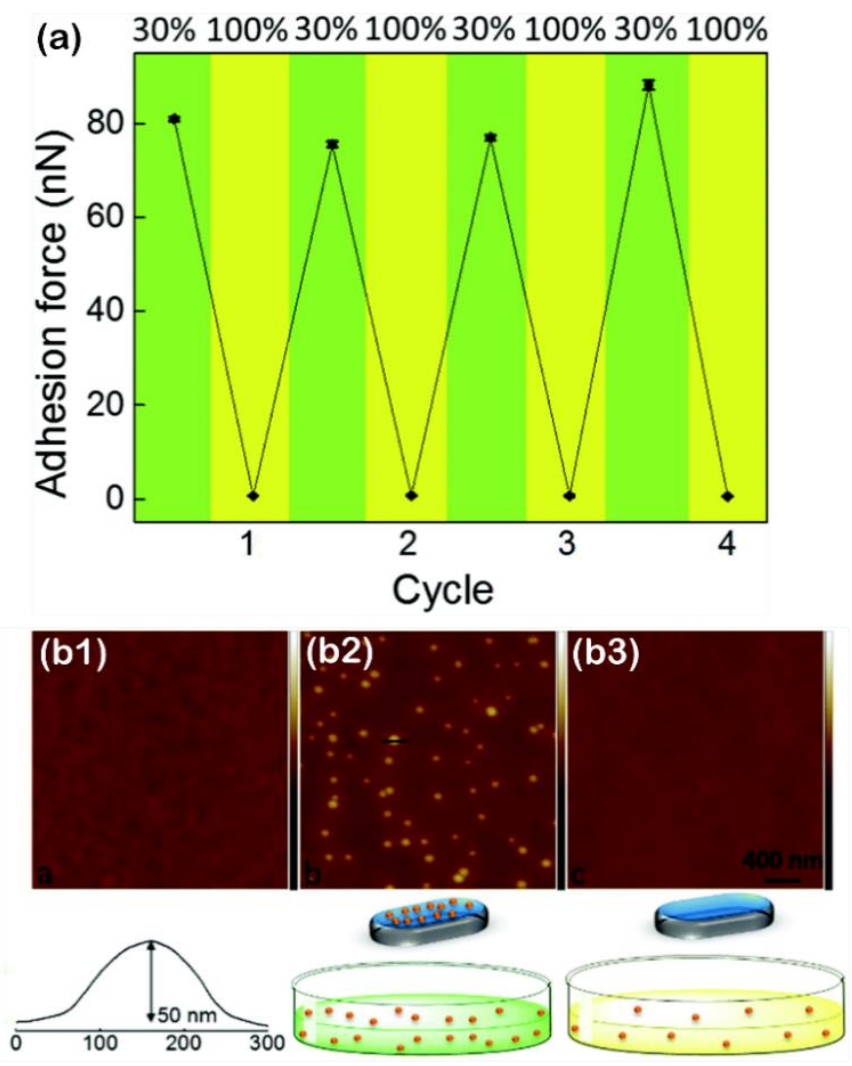

Figure 9 Co-nonsolvency of a PNIPAM brush for the selective pick-up and release of nanoparticles (reproduced with permission from [72]); (a) adhesion force (between a PNIPAM brush and bare silica probe) is switched between pure and 30-70 vol\% ethanol-water mixture. (panel b) Process of extracting the silica nanoparticles; (b1) PNIPAM brush in air, (b2) PNIPAM brush immersed in the nanoparticle solution and dried, (b3) PNIPAM bush immersed in pure ethanol and dried.

Figure 9 (panel b) summarizes the procedure for the extraction and release of silicon nanoparticles using the PNIPAM brush. Accordingly, the particles are extracted in the ethanol-water mixture due to strong particle-polymer adhesion. The AFM image of the brush after immersion in the mixed solvent (Figure 9(b2)) shows the adhered nanoparticles with minor aggregation. Subsequently, ultra-sonication in ethanol removes almost all the adhered particles (Figure 9(b3)) due to the good solvent condition and weak polymer-nanoparticle adhesion.

\section{Conclusions and Outlook}

Thermo-responsive polymer films, in general, can switch between two extreme states: (i) a swollen, highly hydrated, and hydrophilic state; and (ii) a collapsed, less hydrated, and hydrophobic state. As we have 
seen through the reviewed works, the temperature of the aqueous media, solvent composition, and salts can be utilized to manipulate the phase transition of thermo-responsive polymer films. The transition between these two states results in significant changes in the interfacial properties of the film, including the surface forces and friction. With respect to the swollen state, one could typically expect long-range steric repulsive forces, large osmotic effect, high compressibility, weak polymer-polymer and (or) polymer-surface bridging, small adhesion energy, and low friction. Contrarily, the collapsed state is generally represented by relatively short-range steric repulsive forces, low compressibility, strong polymer-polymer and (or) polymer-surface bridging, considerable adhesion energy, and poor lubricity. Besides the general thermo-responsive behaviors, we also highlighted that thin polymer films, more realistically, entail a dense polymer-rich inner region topped with dilute extended tails, which could actively contribute to the surface forces and friction. The outer tails as well as the molecular architecture and conformation of polymer chains, in general, contribute significantly to the surface forces and friction. Furthermore, the way the polymer chains are attached to the substrate and the strength of the polymersurface interface could also influence the surface forces and friction.

On this basis, thermo-responsive polymer films have become attractive candidates for application in the development of smart surfaces with switchable interfacial properties. We have gone through a few examples in Section 3; however, the tuning of the surface forces using thermo-responsive polymer films offers a much broader range of applications. The selective modulation of surface wettability offers great application potential for separation processes, e.g., the fabrication of smart surfaces with "oil-removing" ability at one temperature and "water-removing" ability at another temperature. This functionality requires the surface to reversibly switch between superhydrophobic/superoleophilic and superhydrophilic/superoleophobic states. Herein, a temperature-induced shift in water- and oil-affinity is an effective method that eliminates the need for chemicals. Liquid manipulation is another relevant process, which has diverse applicability in micro/nano fluidic devices, heat transfer, self-lubrication, and biomedical devices. Controlling the liquid spread has been typically conducted through the physical 
patterning of the surface. However, a combination of surface structuring together with thermoresponsivity of surface wettability can provide a better control over droplet spread in a reversible manner. We have partly discussed how thermo-responsive polymer films are employed to modulate the adhesion of living cells to their culture substrate. Based on a similar idea, such coatings can be utilized to fabricate switchable antibacterial surfaces to prevent the proliferation of bacteria and biofilm formation on implants. In this case, surfaces bearing a particular topography or active bactericides, e.g., antibiotics, cationic polymers, and silver ion, can exhibit bactericidal ability. Nevertheless, the continuous contamination by dead bacteria significantly diminishes the bactericidal efficiency. A thermo-responsive polymer film can provide a switchable antifouling feature to the bactericidal surface, meaning that a change in the temperature promotes the detachment of the dead bacteria and could, hence, inhibit biofilm formation. Last but not least, thermo-responsive polymer films can be used for developing smart surfaces with switchable adhesion underwater. This can be done using a combination of mussel-inspired adhesive polymers with a thermo-responsive polymer, where the former serves as the adhesive moiety in water and the latter allows for the reversible modulation of wet adhesion.

Modification of the molecular structure of the polymer chains in order to tune the thermo-responsiveness of the polymer films is an important research area with great practical impact. We have highlighted that a small variation in the chemistry of the polymer backbone, e.g., addition of a more hydrophilic comonomer, can significantly affect the thermo-responsive behavior of the film in the terms of shifting the transition temperature as well as modifying the volume phase transition. On the other hand, modifying the molecular architecture of the polymer chains to obtain a desired conformation at the film interface is another essential research direction. We have seen that the dilute tails at the film periphery notably affect the interfacial properties, e.g., adhesiveness and lubricity, and their thermo-responsivity. To address this issue, one can tune the molecular architecture through different approaches: (i) modifying the intrinsic structural properties of the polymer such as polydispersity, (ii) design of more complex architectures rather than linear homopolymers, e.g., cyclic polymers, and (iii) design of novel block 
copolymers and bottlebrush polymers with optimized chemistry and architecture. Finally, multicomponent polymer systems allow for further tuning the interfacial properties and thermoresponsiveness through synergistic effects in terms of improving the attachment to the surface, enhancing the cohesion within the film, modifying the hydration levels, and controlling the interfacial conformation.

\section{Acknowledgments}

We wish to thank the Innovation Fund Denmark for financial support through the Grand Solutions Project \#6151-00007B

\section{References}

[1] Badi N. Non-linear PEG-based thermoresponsive polymer systems. Prog Polym Sci 2017;66:54-79. https://doi.org/10.1016/j.progpolymsci.2016.12.006.

[2] Nagase K, Yamato M, Kanazawa H, Okano T. Poly(N-isopropylacrylamide)-based thermoresponsive surfaces provide new types of biomedical applications. Biomaterials 2018;153:27-48. https://doi.org/10.1016/j.biomaterials.2017.10.026.

[3] Liu X, Lin W, Astruc D, Gu H. Syntheses and applications of dendronized polymers. Prog Polym Sci 2019;96:43-105. https://doi.org/10.1016/j.progpolymsci.2019.06.002.

[4] Dai Y, Chen X, Zhang X. Recent advances in stimuli-responsive polymeric micelles via click chemistry. Polym Chem 2018;10:34. https://doi.org/10.1039/c8py01174e.

[5] Kauscher U, Holme MN, Björnmalm M, Stevens MM. Physical stimuli-responsive vesicles in drug delivery: Beyond liposomes and polymersomes. Adv Drug Deliv Rev 2019;138:259-75. https://doi.org/10.1016/j.addr.2018.10.012.

[6] Hu X, Zhang Y, Xie Z, Jing X, Bellotti A, Gu Z. Stimuli-Responsive Polymersomes for Biomedical Applications. Biomacromolecules 2017;18:649-73. https://doi.org/10.1021/acs.biomac.6b01704.

[7] Kim YJ, Matsunaga YT. Thermo-responsive polymers and their application as smart biomaterials. J Mater Chem B 2017;5:4307-21. https://doi.org/10.1039/c7tb00157f.

[8] Demirci S, Kinali-Demirci S, Jiang S. A switchable polymer brush system for antifouling and controlled detection. Chem Commun 2017;53:3713-6. https://doi.org/10.1039/c7cc00193b.

[9] Liu J, Wang N, Yu LJ, Karton A, Li W, Zhang W, et al. Bioinspired graphene membrane with temperature tunable channels for water gating and molecular separation. Nat Commun 2017; 8:2011. https://doi.org/10.1038/s41467-017-02198-5. 

Broad Temperature Region Realized by Cross-Linking Thermoresponsive Microgels onto Cotton Fabrics. ACS Appl Mater Interfaces 2019;11:30269-77. https://doi.org/10.1021/acsami.9b09294.

[11] Fu W, Bai W, Jiang S, Seymour BT, Zhao B. UCST-Type Thermoresponsive Polymers in Synthetic Lubricating Oil Polyalphaolefin (PAO). Macromolecules 2018;51:1674-80. https://doi.org/10.1021/acs.macromol.7b02755.

[12] Murdoch TJ, Humphreys BA, Johnson EC, Webber GB, Wanless EJ. Specific ion effects on thermoresponsive polymer brushes: Comparison to other architectures. J Colloid Interface Sci 2018;526:429-50. https://doi.org/10.1016/j.jcis.2018.04.086. * This review study provides comprehensive information of thermo-responsive properties and the effects of salts on polymer brushes.

[13] Halperin A, Kröger M, Winnik FM. Poly ( N -isopropylacrylamide) Phase Diagrams: Fifty Years of Research. Angew Chemie Int Ed 2015;54:15342-67. https://doi.org/10.1002/anie.201506663.

[14] Liu X, Yang Y, Urban MW. Stimuli-Responsive Polymeric Nanoparticles. Macromol Rapid Commun 2017;38:1700030. https://doi.org/10.1002/marc.201700030.

[15] Ma S, Zhang X, Yu B, Zhou F. Brushing up functional materials. NPG Asia Mater 2019;11:24. https://doi.org/10.1038/s41427-019-0121-2.

[16] Moad G. RAFT polymerization to form stimuli-responsive polymers. Polym Chem 2017;8:177-219. https://doi.org/10.1039/C6PY01849A.

[17] Matyjaszewski K. Advanced Materials by Atom Transfer Radical Polymerization. Adv Mater 2018;30:1706441. https://doi.org/10.1002/adma.201706441. Zhou T, Qi H, Han L, Barbash D, Li CY. Towards controlled polymer brushes via a self-assembly-assisted-grafting-to approach. Nat Commun 2016;7:11119. https://doi.org/10.1038/ncomms11119.

[19] Blasco E, Sims MB, Goldmann AS, Sumerlin BS, Barner-Kowollik C. 50th Anniversary Perspective: Polymer Functionalization. Macromolecules 2017;50:5215-52. https://doi.org/10.1021/acs.macromol.7b00465.

Faivre J, Shrestha BR, Xie G, Olszewski M, Adibnia V, Moldovan F, et al. Intermolecular interactions between bottlebrush polymers boost the protection of surfaces against frictional wear. Chem Mater 2018;30:4140-9. https://doi.org/10.1021/acs.chemmater.8b01676.

[21] Netz RR, Andelman D. Neutral and charged polymers at interfaces. Phys Rep 2003;380:1-95. https://doi.org/10.1016/S0370-1573(03)00118-2.

[22] Ballauff M, Borisov O V. Phase transitions in brushes of homopolymers. Polymer 2016;98:402-8. ttps://doi.org/10.1016/j.polymer.2016.05.014.

[23] Zhuang P, Dirani A, Glinel K, Jonas AM. Temperature Dependence of the Surface and Volume Hydrophilicity of Hydrophilic Polymer Brushes. Langmuir 2016;32:3433-44. https://doi.org/10.1021/acs.langmuir.6b00448. * This work investigated the thermo-responsive behavior of polymer films in terms of surface and volume phase transitions.

[24] Kooij ES, Sui X, Hempenius MA, Zandvliet HJW, Vancso GJ. Probing the Thermal Collapse of Poly( N -isopropylacrylamide) Grafts by Quantitative in Situ Ellipsometry. J Phys Chem B 2012;116:9261-8. https://doi.org/10.1021/jp304364m.

[25] Varma S, Bureau L, Débarre D. The Conformation of Thermoresponsive Polymer Brushes Probed by Optical Reflectivity. Langmuir 2016;32:3152-63. https://doi.org/10.1021/acs.langmuir.6b00138. 

ellipsometry-study of temperature-responsive polymer brush systems. Appl Surf Sci 2017;421:843-51.

https://doi.org/10.1016/j.apsusc.2017.02.078. * This work investigates the thermo-responsive behavior of PNIPAM brush using QCM-D and ellipsometry; and discusses the differences in the outcome of the two methods.

[27] Humphreys BA, Prescott SW, Murdoch TJ, Nelson A, Gilbert EP, Webber GB, et al. Influence of molecular weight on PNIPAM brush modified colloidal silica particles. Soft Matter 2019;15:55-64. https://doi.org/10.1039/C8SM01824C.

Koenig M, Rodenhausen KB, Rauch S, Bittrich E, Eichhorn K-J, Schubert M, et al. Salt Sensitivity of the Thermoresponsive Behavior of PNIPAAm Brushes. Langmuir 2018;34:2448-54. https://doi.org/10.1021/acs.langmuir.7b03919.

Myshkin N, Kovalev A. Adhesion and surface forces in polymer tribology—A review. Friction 2018;6:143-55. https://doi.org/10.1007/s40544018-0203-0.

[30] Mocny P, Klok H-A. Tribology of surface-grafted polymer brushes. Mol Syst Des Eng 2016;1:141-54. https://doi.org/10.1039/C5ME00010F.

[31] Feng L, Laderman B, Sacanna S, Chaikin P. Re-entrant solidification in polymer-colloid mixtures as a consequence of competing entropic and enthalpic attractions. Nat Mater 2015;14:61-5. https://doi.org/10.1038/nmat4109.

[32] Liang X, Nakajima K. Nanofishing of a Single Polymer Chain: Temperature-Induced Coil-Globule Transition of Poly( N -isopropylacrylamide) Chain in Water. Macromol Chem Phys 2018;219:1700394. https://doi.org/10.1002/macp.201700394.

[33] Xie F, Woodward CE, Forsman J. Non-monotonic temperature response of polymer mediated interactions. Soft Matter 2016;12:658-63. https://doi.org/10.1039/C5SM02420J.

[34] Chen H, Cox JR, Ow H, Shi R, Panagiotopoulos AZ. Hydration Repulsion between Carbohydrate Surfaces Mediated by Temperature and Specific Ions. Sci Rep 2016;6:28553. https://doi.org/10.1038/srep28553.

[35] An J, Liu X, Dedinaite A, Korchagina E, Winnik FM, Claesson PM. Effect of solvent quality and chain density on normal and frictional forces between electrostatically anchored thermoresponsive diblock copolymer layers. J Colloid Interface Sci 2017;487:88-96. https://doi.org/10.1016/j.jcis.2016.10.021.

[36] Wassel E, Wesner D, Schönherr H. Colloidal force probe study of poly(di(ethylene glycol)methylether methacrylate) homopolymer brush layers in aqueous media at different temperatures. Eur Polym J 2017;89:440-8. https://doi.org/10.1016/j.eurpolymj.2017.02.038.

[37] Mathis CH, Divandari M, Simic R, Naik V V, Benetti EM, Isa L, et al. ATR-IR Investigation of Solvent Interactions with Surface-Bound Polymers. Langmuir 2016;32:7588-95. https://doi.org/10.1021/acs.langmuir.6b02086.

[38] Yu Y, Kieviet BD, Kutnyanszky E, Vancso GJ, de Beer S. Cosolvency-Induced Switching of the Adhesion between Poly(methyl methacrylate) Brushes. ACS Macro Lett 2015;4:75-9. https://doi.org/10.1021/mz500775w.

[39] Yu Y, Kieviet BD, Liu F, Siretanu I, Kutnyánszky E, Vancso GJ, et al. Stretching of collapsed polymers causes an enhanced dissipative response of PNIPAM brushes near their LCST. Soft Matter 2015;11:8508-16. https://doi.org/10.1039/c5sm01426c.

[40] De Beer S, Kutnyanszky E, Schön PM, Vancso GJ, Müser MH. Solvent-induced immiscibility of polymer brushes eliminates dissipation 
channels. Nat Commun 2014;5:3781. https://doi.org/10.1038/ncomms4781.

[41] Chen Q, Kooij ES, Sui X, Padberg CJ, Hempenius MA, Schön PM, et al. Collapse from the top: Brushes of poly(N-isopropylacrylamide) in cononsolvent mixtures. Soft Matter 2014;10:3134-42. https://doi.org/10.1039/c4sm00195h.

[42] Dehghani ES, Spencer ND, Ramakrishna SN, Benetti EM. Crosslinking polymer brushes with ethylene glycol-containing segments: Influence on physicochemical and antifouling properties. Langmuir 2016;32:10317-27. https://doi.org/10.1021/acs.langmuir.6b02958.

[43] Synytska A, Biehlig E, Ionov L. Adaptive PEG-PDMS Brushes: Effect of architecture on adhesiveness in air and under water. Macromolecules 2014;47:8377-85. https://doi.org/10.1021/ma501968y.

[44] Divandari M, Morgese G, Ramakrishna SN, Benetti EM. Surface-grafted assemblies of cyclic polymers: Shifting between high friction and extreme lubricity. Eur Polym J 2019;110:301-6. https://doi.org/10.1016/j.eurpolymj.2018.11.039.

[45] Faivre J, Shrestha BR, Xie G, Delair T, David L, Matyjaszewski K, et al. Unraveling the Correlations between Conformation, Lubrication, and Chemical Stability of Bottlebrush Polymers at Interfaces. Biomacromolecules 2017;18:4002-10. https://doi.org/10.1021/acs.biomac.7b01063.

[46] Faivre J, Shrestha BR, Burdynska J, Xie G, Moldovan F, Delair T, et al. Wear Protection without Surface Modification Using a Synergistic Mixture of Molecular Brushes and Linear Polymers. ACS Nano 2017;11:1762-9. https://doi.org/10.1021/acsnano.6b07678.

[47] Shoaib T, Heintz J, Lopez-Berganza JA, Muro-Barrios R, Egner SA, Espinosa-Marzal RM. Stick-Slip Friction Reveals Hydrogel Lubrication Mechanisms. Langmuir 2018;34:756-65. https://doi.org/10.1021/acs.langmuir.7b02834.

[48] Benetti EM, Spencer ND. Using Polymers to Impart Lubricity and Biopassivity to Surfaces: Are These Properties Linked? Helv Chim Acta 2019;102:e1900071. https://doi.org/10.1002/hlca.201900071.

[49] Dedinaite A, Claesson PM. Synergies in lubrication. Phys Chem Chem Phys 2017;19:23677-89. https://doi.org/10.1039/C7CP03517A.

[50] Ramakrishna SN, Cirelli M, Divandari M, Benetti EM. Effects of Lateral Deformation by Thermoresponsive Polymer Brushes on the Measured Friction Forces. Langmuir 2017;33:4164-71. https://doi.org/10.1021/acs.langmuir.7b00217. * The authors in this work discuss how polymer chains under shear can laterally deform depending on the film thickness and sliding distance. Temperature, on the other hand, has a significant effect on this process, as collapsed chains resist stretching.

[51] Graham S, Marina PF, Blencowe A. Thermoresponsive polysaccharides and their thermoreversible physical hydrogel networks. Carbohydr Polym 2019;207:143-59. https://doi.org/10.1016/j.carbpol.2018.11.053.

[52] Thormann E, Bodvik R, Karlson L, Claesson PM. Surface forces and friction between non-polar surfaces coated by temperature-responsive methylcellulose. Colloids Surfaces A Physicochem Eng Asp 2014;441:701-8. https://doi.org/10.1016/j.colsurfa.2013.10.038.

[53] Dobryden I, Cortes Ruiz M, Zhang X, Dedinaite A, Wieland DCF, Winnik FM, et al. Thermoresponsive Pentablock Copolymer on Silica: Temperature Effects on Adsorption, Surface Forces, and Friction. Langmuir 2019;35:653-61. https://doi.org/10.1021/acs.langmuir.8b03729. * The authors in this work studied a pentablock copolymer that upon adsorption to silica surface creates thermo-responsive layers. The provided discussions on surface forces and firction give a better insight on how polymer-surface and polymer-polymer interactions affect lubrcity and load-bearing ability. 

2002;106:3827-34. https://doi.org/10.1021/jp013199q.

[55] Yan W, Ramakrishna SN, Spencer ND, Benetti EM. Brushes, Graft Copolymers, or Bottlebrushes? The Effect of Polymer Architecture on the Nanotribological Properties of Grafted-from Assemblies. Langmuir 2019;35:11255-64. https://doi.org/10.1021/acs.langmuir.9b01265.

[56] Morgese G, Gombert Y, Ramakrishna SN, Benetti EM. Mixing Poly(ethylene glycol) and Poly(2-alkyl-2-oxazoline)s Enhances Hydration and Viscoelasticity of Polymer Brushes and Determines Their Nanotribological and Antifouling Properties. ACS Appl Mater Interfaces 2018;10:41839-48. https://doi.org/10.1021/acsami.8b17193. * this study demonstrates how hydration and surface forces in thermo-responsive mixed brushes can provide enhanced lubrication and antifouling properties.

An J, Liu X, Linse P, Dèdinaite A, Winnik FM, Claesson PM. Tethered Poly(2-isopropyl-2-oxazoline) Chains: Temperature Effects on Layer Structure and Interactions Probed by AFM Experiments and Modeling. Langmuir 2015;31:3039-48. https://doi.org/10.1021/la504653w.

[58] Zajforoushan Moghaddam S, Zhu K, Nyström B, Thormann E. Thermo-responsive diblock and triblock cationic copolymers at the silica/aqueous interface: A QCM-D and AFM study. J Colloid Interface Sci 2017;505:546-55. https://doi.org/10.1016/j.jcis.2017.06.044. ** This study investigates the surface forces on triblock copolymers with thermo-responsive behavior. The copolymer contains two thermoresponsive units where one controls the inner structure and the other controls the outer properties of the film. Murdoch TJ, Humphreys BA, Johnson EC, Prescott SW, Nelson A, Wanless EJ, et al. The role of copolymer composition on the specific ion and thermo-response of ethylene glycol-based brushes. Polymer 2018;138:229-41. https://doi.org/10.1016/j.polymer.2018.01.053. ** The authors in this study examined the surface forces in ethylene glycol-based brushes with different chemical structures. It is shown how a slight change in the structure affects the surface forces and viscoelastic properties of the brush.

Zajforoushan Moghaddam S, Thormann E. Hofmeister effect of salt mixtures on thermo-responsive poly(propylene oxide). Phys Chem Chem Phys 2015;17:6359-66. https://doi.org/10.1039/C4CP05677A.

[61] Moghaddam SZ, Thormann E. Hofmeister effect on thermo-responsive poly(propylene oxide): Role of polymer molecular weight and concentration. J Colloid Interface Sci 2016;465:65-75. https://doi.org/10.1016/j.jcis.2015.11.040. 35. https://doi.org/10.1016/j.jcis.2019.07.067.

[63] Murdoch TJ, Humphreys BA, Willott JD, Gregory KP, Prescott SW, Nelson A, et al. Specific Anion Effects on the Internal Structure of a Poly( N -isopropylacrylamide) Brush. Macromolecules 2016;49:6050-60. https://doi.org/10.1021/acs.macromol.6b01001.

[64] Tang Y, Zhang X, Choi P, Liu Q, Xu Z. Probing Single-Molecule Adhesion of a Stimuli Responsive Oligo(ethylene glycol) Methacrylate Copolymer on a Molecularly Smooth Hydrophobic MoS 2 Basal Plane Surface. Langmuir 2017;33:10429-38. https://doi.org/10.1021/acs.langmuir.7b01187.

[65] Zajforoushan Moghaddam S, Thormann E. Hofmeister Effect on PNIPAM in Bulk and at an Interface: Surface Partitioning of Weakly Hydrated Anions. Langmuir 2017;33:4806-15. https://doi.org/10.1021/acs.langmuir.7b00953. ** This work provides information on thermo-responsive properties of PNIPAM in saline solutions both in bulk and at interface. The surface forces measurements demonstrate how polymer interactions 
are tuned i the presence of different salts.

[66] Humphreys BA, Johnson EC, Wanless EJ, Webber GB. Poly( N -isopropylacrylamide) Response to Salt Concentration and Anion Identity: A Brush-on-Brush Study. Langmuir 2019;35:10818-30. https://doi.org/10.1021/acs.langmuir.9b00695. ** This work investigates the effect of salts on polymer-polymer and polymer-surface interactions, discussing the steric and electrostatic effects on the surface forces.

[67] Murdoch TJ, Humphreys BA, Willott JD, Prescott SW, Nelson A, Webber GB, et al. Enhanced specific ion effects in ethylene glycol-based thermoresponsive polymer brushes. J Colloid Interface Sci 2017;490:869-78. https://doi.org/10.1016/j.jcis.2016.11.044.

[68] Stöbener DD, Scholz J, Schedler U, Weinhart M. Switchable Oligo(glycidyl ether) Acrylate Bottlebrushes “Grafted-from” Polystyrene Surfaces: A Versatile Strategy toward Functional Cell Culture Substrates. Biomacromolecules 2018;19:4207-18. https://doi.org/10.1021/acs.biomac.8b00933.

[69] Chen L, Xie Z, Gan T, Wang Y, Zhang G, Mirkin CA, et al. Biomimicking Nano-Micro Binary Polymer Brushes for Smart Cell Orientation and Adhesion Control. Small 2016;12:3400-6. https://doi.org/10.1002/smll.201600634.

[70] Akiyama Y, Matsuyama M, Yamato M, Takeda N, Okano T. Poly(N-isopropylacrylamide)-Grafted Polydimethylsiloxane Substrate for Controlling Cell Adhesion and Detachment by Dual Stimulation of Temperature and Mechanical Stress. Biomacromolecules 2018;19:4014-22. https://doi.org/10.1021/acs.biomac.8b00992. * This work demonstrates that changes in the surface adhesion of PNIPAM brushes can promote attachment/detachment of living cells on top. In addition, it is discussed how thickness and dilute tails can modulate the adhesiveness.

[71] Ye Y, Huang J, Wang X. Fabrication of a Self-Cleaning Surface via the Thermosensitive Copolymer Brush of P(NIPAAm-PEGMA). ACS Appl Mater Interfaces 2015;7:22128-36. https://doi.org/10.1021/acsami.5b07336. * In this work, a copolymer with optimized chemical composition is prepared that shows oil-repellency and self-cleaning properties. The transition in surface hydrophobicity as well as sudden volume transition promote self-cleaning.

[72] Yu Y, Lopez de la Cruz RA, Kieviet BD, Gojzewski H, Pons A, Julius Vancso G, et al. Pick up, move and release of nanoparticles utilizing conon-solvency of PNIPAM brushes. Nanoscale 2017;9:1670-5. https://doi.org/10.1039/C6NR09245D. * This work uses co-nonsolvency on polymer brushes as a way to tune the surface adhesion. Based on this, the polymer film is used to selectively capture nanoparticles from one solution and release in anot 